\title{
Nonlinear Dynamic Inversion Baseline Control Law: Flight-Test Results for the Full-scale Advanced Systems Testbed F/A-18 Airplane
}

\author{
Christopher J. Miller ${ }^{1}$ \\ NASA Dryden Flight Research Center, Edwards, California, 93534
}

\begin{abstract}
A model reference nonlinear dynamic inversion control law has been developed to provide a baseline controller for research into simple adaptive elements for advanced flight control laws. This controller has been implemented and tested in a hardware-in-the-loop simulation and in flight. The flight results agree well with the simulation predictions and show good handling qualities throughout the tested flight envelope with some noteworthy deficiencies highlighted both by handling qualities metrics and pilot comments. Many design choices and implementation details reflect the requirements placed on the system by the nonlinear flight environment and the desire to keep the system as simple as possible to easily allow the addition of the adaptive elements. The flight-test results and how they compare to the simulation predictions are discussed, along with a discussion about how each element affected pilot opinions. Additionally, aspects of the design that performed better than expected are presented, as well as some simple improvements that will be suggested for follow-on work.
\end{abstract}

\section{Nomenclature}

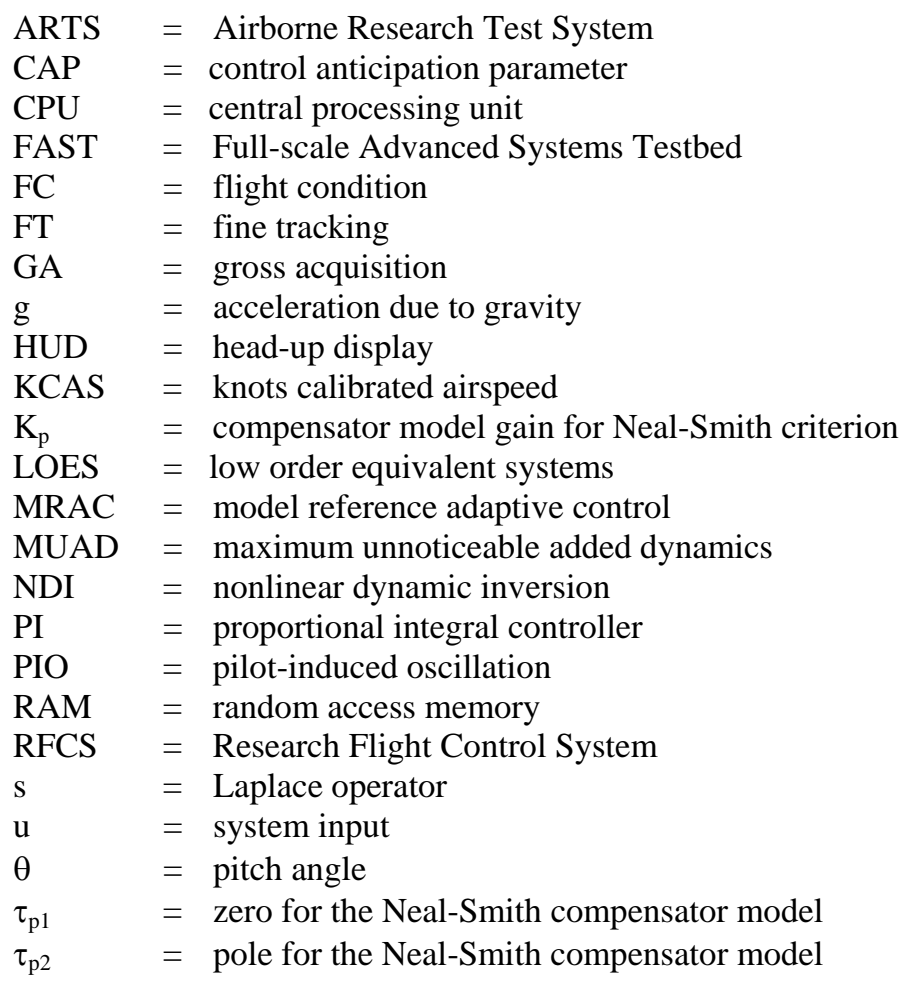

\footnotetext{
${ }^{1}$ Research Engineer, Flight Controls and Dynamics, P.O. Box 273, MS 4840D, Edwards, California 93523, AIAA Member.
} 


\section{Introduction}

$\mathrm{N}$

onlinear dynamic inversion (NDI) as a control architecture has been investigated for years and it is now being applied to new production vehicles. ${ }^{1,2}$ Therefore, the intent of this paper is not to prove the viability of this type of control scheme, but to discuss implementation details for a simple NDI control law for the Full-scale Advanced Systems Testbed (FAST) F/A-18 airplane (McDonnell Douglas, now The Boeing Company, Chicago, Illinois). The control law is hosted in a research processor with build-in protection restricting the available envelope. This architecture is designed specifically to be a baseline controller upon which advanced control elements can be easily added and will enable further control research into adaptive controls ${ }^{3-10}$ and the control of flexible structures. ${ }^{11,12}$ The NDI control law is a first step toward building a working environment in which design changes and new research objectives can be quickly brought to flight and their real behavior ascertained. The choice of dynamic inversion was driven by the mathematically and kinematically intuitive architecture, explicit model-following behavior, the ability to be used to introduce fundamental-level simulated failures within the aerodynamic model for testing the performance of advanced control elements, and because it can be included in the stability proofs for many advanced control schemes. ${ }^{3}$

The focus of this paper is to present the flight-test results for the baseline NDI control law design outlined in Ref. 13 with special attention given to the comparisons between the simulation predictions and flight. Additionally, aspects of the design that performed better than expected are presented, as well as some simple improvements that will be suggested for follow-on work. Flight data are evaluated against the same handling qualities metrics used in design ${ }^{13}$ and flight-determined stability margins are presented. Finally, pilot comments and ratings for two closed-loop tracking tasks are presented, and are considered the final necessary piece of the data required to definitively ascertain the actual handling qualities for the NDI / F-18 system and highlight areas where improvements are warranted.

\section{Background}

The control law that is the subject of this paper has been designed to be an available baseline control law for the FAST platform. The FAST platform is fundamentally a single-seat F/A-18 airplane, as shown in Fig. 1 and Fig. 2. Substantial research instrumentation (structural, air data, and inertial) was installed on this airplane to support the Active Aeroelastic Wing (AAW) program. ${ }^{14}$ The robust nature of the testbed (structural load capacity, spin and recovery characteristics, and reversion to production control laws), along with the research instrumentation, enable flight-testing of novel control laws with minimal validation testing requirements for a piloted flight vehicle.

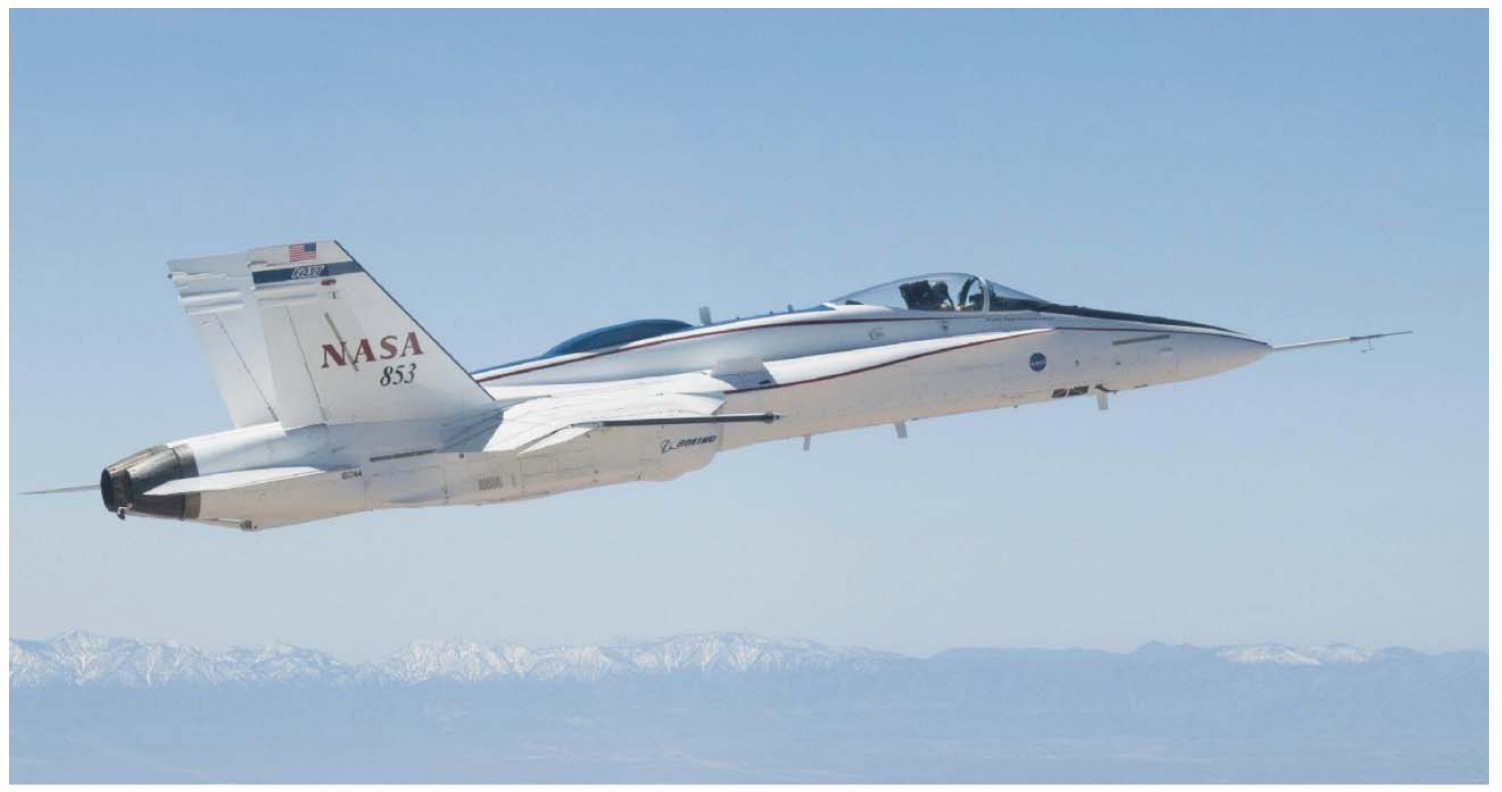

Figure 1. The Full-scale Advanced Systems Testbed F/A-18 airplane in flight. 


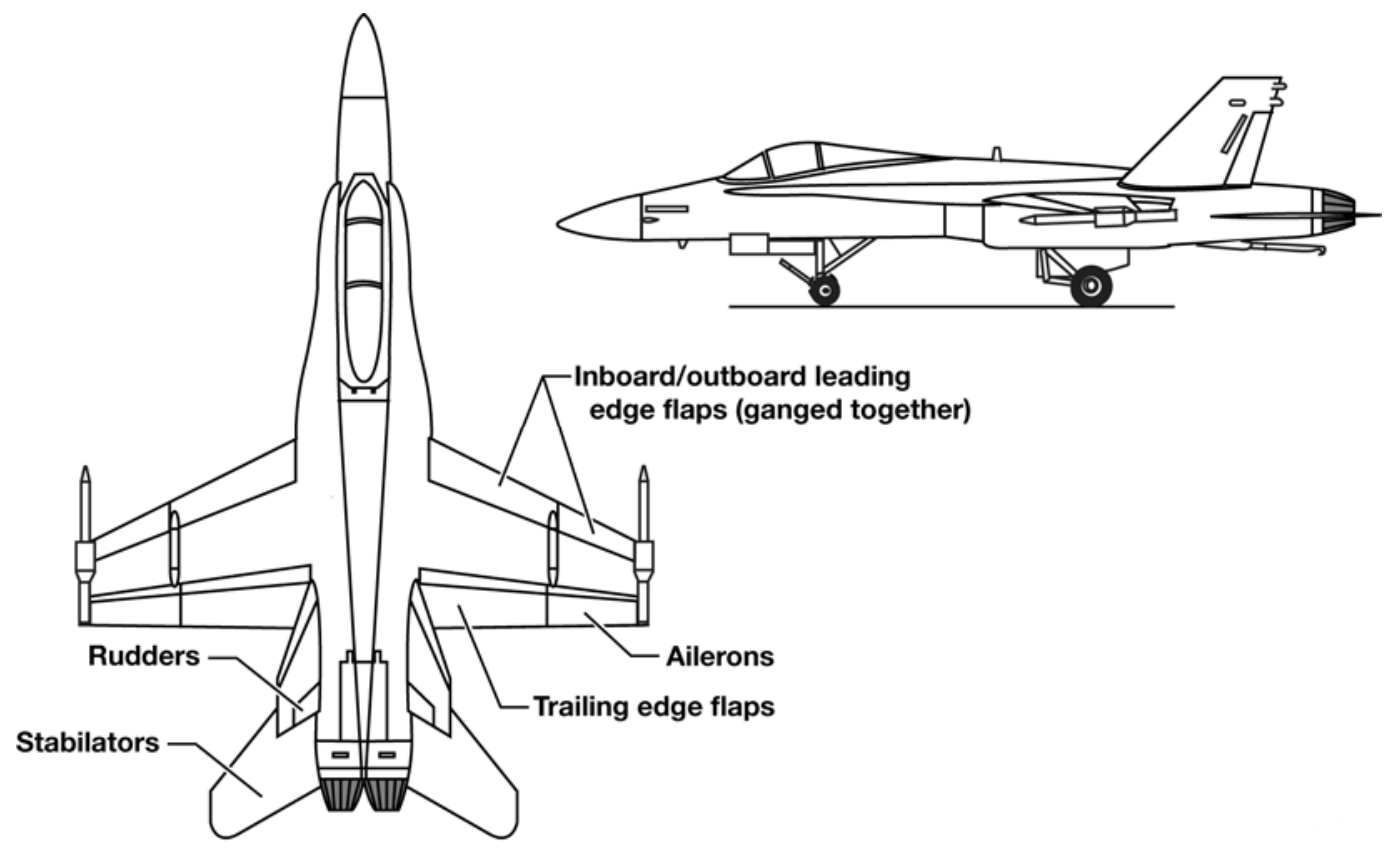

Figure 2. The control surfaces of the F/A-18 airplane.

Figure 3 shows the control computer architecture for FAST. The system maintains the advantages of the production system and utilizes its redundancy management architecture for sensor selection and actuator signal management. The FAST research flight control computer architecture, which builds upon legacy F/A-18 research systems, consists of two separate research processing capabilities. The Research Flight Control System (RFCS) provides a minimal delay quad-redundant processing environment in which Ada-programmed experiments can be executed. The RFCS also performs some envelope protection for restricting where a given research control law hosted in either the Airborne Research Test System (ARTS) or RFCS can remain engaged, as shown in Fig. 4. The ARTS, with its more capable processor and the ability to host Simulink ${ }^{\circledR}$ (The MathWorks, Natick, Massachusetts) autocode or $\mathrm{C}$ code provides a more flexible environment for novel control laws than does the RFCS; however, the ARTS is only dual-redundant, and imparts one additional frame of delay to commands. This extra frame of delay translates to 0.0125 seconds for the pitch and roll axes and 0.025 seconds for the yaw axis. 


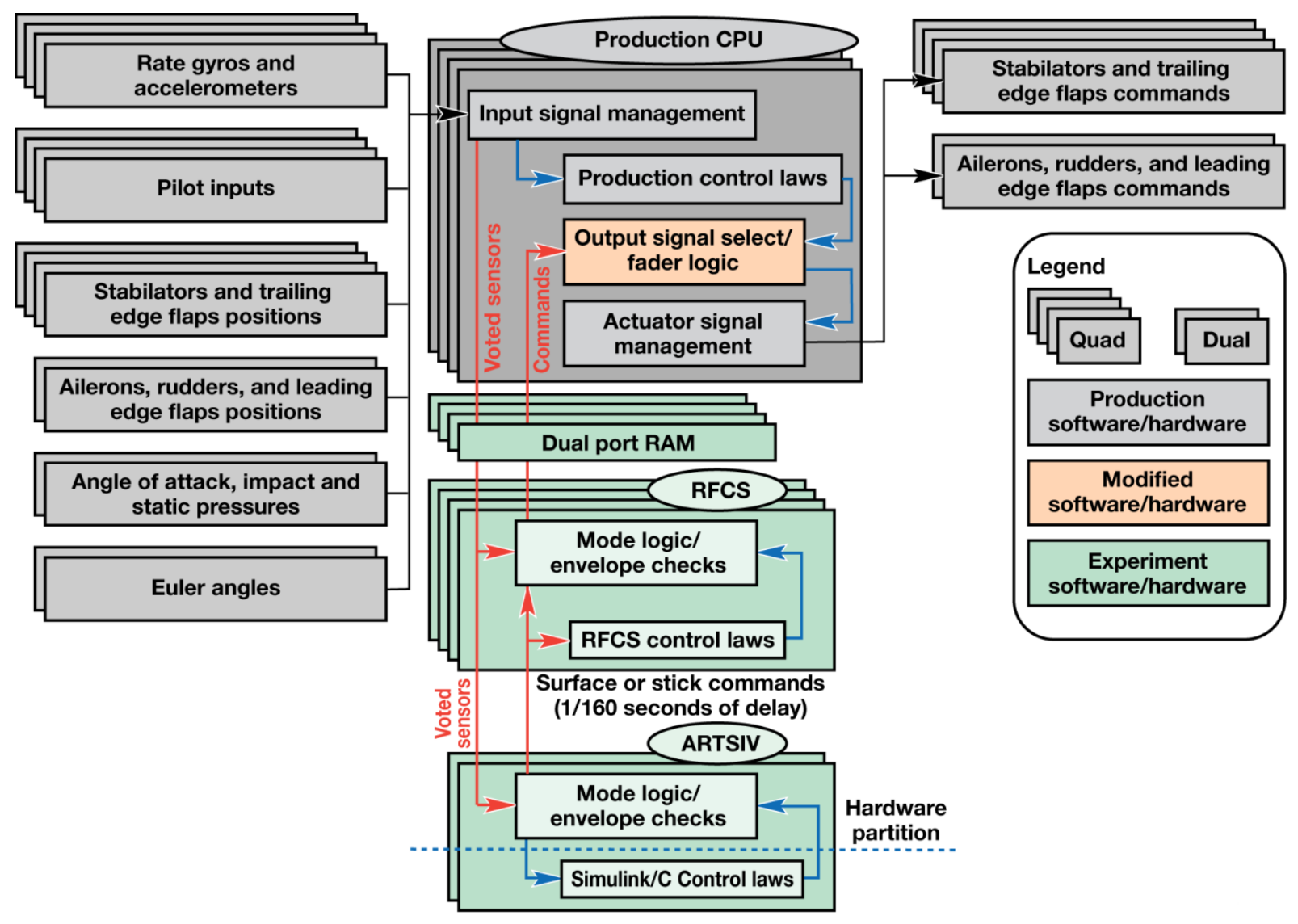

Figure 3. The Full-scale Advanced Systems Testbed Research Flight Control System Airborne Research Test System control computer architecture.

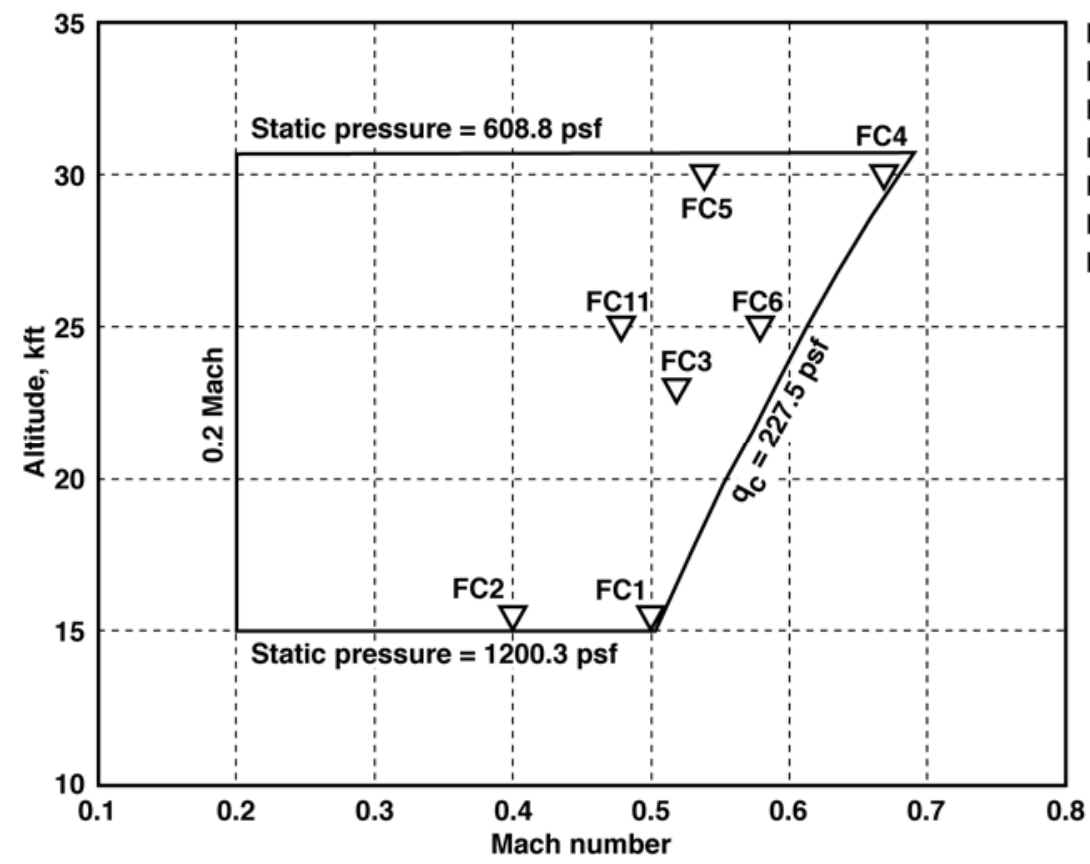

FC1: 250 KCAS, 15.5 kft

FC2: $200 \mathrm{KCAS}, 15.5 \mathrm{kft}$

FC3: 223 KCAS, $23.0 \mathrm{kft}$

FC4: 250 KCAS, 30.0 kft

FC5: $200 \mathrm{KCAS}, 30.0 \mathrm{kft}$

FC6: 240 KCAS, $25.0 \mathrm{kft}$

FC11: $200 \mathrm{KCAS}, 25.0 \mathrm{kft}$

Figure 4. The Full-scale Advanced Systems Testbed flight envelope and flight conditions. 
The FAST research system has three operational modes: disengaged, armed, and engaged. Data passes between the production and research processors in all three modes, but research control laws are only in control of the aircraft in the engaged state. The pilot can disengage the system at any time using the autopilot disengage switch, and return control to the production control laws. This architecture provides researchers the opportunity to observe the research control law behavior in the disengaged and armed states prior to engaging it, and allows the reversion to the well-known and robust production control law in the event that the research control law exhibits undesirable behavior.

In addition to the flight assets, a simulation facility provides a hardware-in-the-loop environment for design and testing of new control techniques. The facility consists of an F/A-18 test bench with flight control hardware, a full nonlinear simulation environment, and hardware ARTS units. Without this facility, rapid design and prototyping would not be possible.

\section{Control Law Description}

The NDI control law considered here was implemented in the ARTS and contains a number of distinct components, as depicted in Fig. 5, each with their own design goals and functions. At the core of the control law is the actual dynamic inversion, which computes the surface positions necessary to achieve the desired aircraft dynamics using the equations of motion and, in this case, simplified aerodynamic tables from the simulation. These desired aircraft dynamics are computed from the pilot stick commands via the use of transfer-function-based reference models. The goal is to give the pilot the type of vehicle response desired and expected; however, as with any real system, the model used in the inversion cannot be expected to exactly predict the actual behavior of the vehicle. Therefore, a compensator must be added to provide the necessary robustness to these modeling inaccuracies. This compensation is accomplished by adding a proportional-plus-integral compensator that is intended to drive down the error between the desired dynamics and the actual dynamics. Structural filters are also needed to attenuate the structural vibration from the feedback sensors to prevent any kind of undesirable aeroservoelastic interactions. This basic and simple architecture was chosen based on its applicability to adaptive flight control research; ${ }^{6,7}$ however, it need not be limited to adaptive control research. ${ }^{11,}{ }^{12}$ Reference 13 contains a detailed description of the control law under test, and presents the simulation performance predictions.

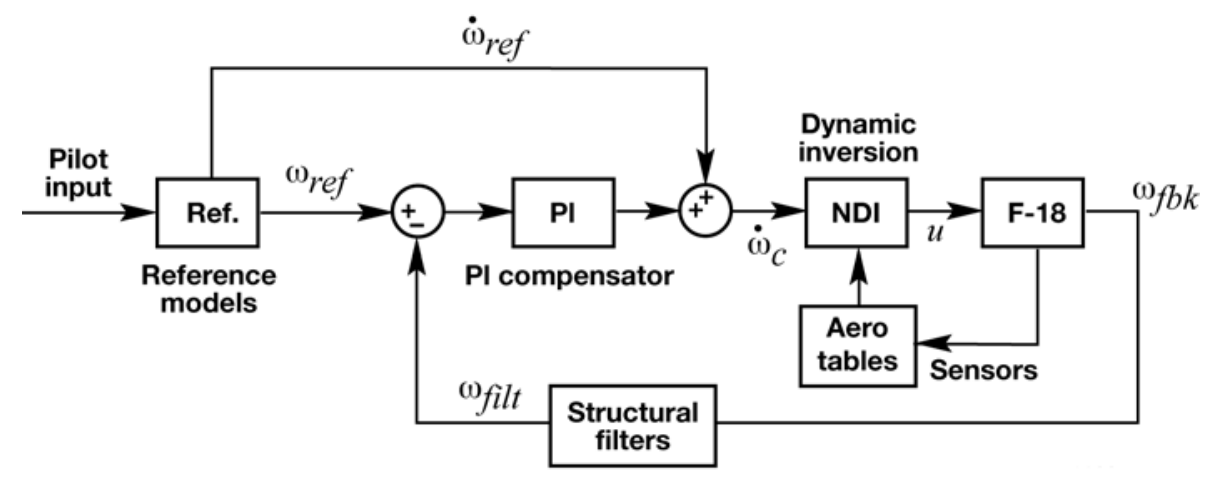

Figure 5. Block diagram of the nonlinear dynamic inversion control law.

\section{Development Schedule}

An aggressive development schedule was executed, enabled by the fact that the test system architecture was designed with rapid prototyping in mind. Table 1 shows some of the important milestones for the NDI development. It is worth noting that the NDI was the first closed-loop control law designed and implemented by the test team. Despite the team's relative inexperience with the asset, the NDI was taken from concept to flight in a little over four months, completing verification and validation testing in just under one month. This kind of aggressive schedule is facilitated by the design of the fourth-generation ARTS (ARTS IV) and RFCS architecture, and the fact that the F/A-18 airplane is a robust vehicle especially when operated within the envelope shown in Fig. 4. 
Table 1. The development schedule for nonlinear dynamic inversion.

Concept preliminary design review

Start software verification and validation with NDI version 1.0

$8 / 16 / 2010$

Complete software verification and validation with NDI version 1.4

9/10/2010

First flight version 1.4 (flight 112)

9/27/2010

First flight version 1.5 (flight 114)

$10 / 22 / 2010$

Final NDI flight (flight 117)

$11 / 29 / 2010$

\section{Flight-Test Results}

A total of six research flights were flown with the baseline NDI control law at various flight conditions (FCs) within the envelope shown in Fig. 4. Three test pilots accumulated approximately five total flight hours on the control law. This limited number of flight hours is in no way sufficient to definitively state that the control law has been fully explored; however, a number of different maneuvers and FCs were tested to highlight the strengths and weaknesses of the NDI. These maneuvers included: three-axis frequency sweeps, doublets, bank captures, steady-heading sideslips, $360^{\circ}$ rolls, wind-up turns, loaded rolls, air-to-air tracking, and in-trail formation flight. The following discussions highlight the findings of the limited flight-testing performed on the control law, along with comparisons to the predictions from the simulation. ${ }^{13}$

\section{A. Simulation-to-Flight Comparisons and Handling Qualities Metrics}

The nonlinear simulation was used to evaluate the design both from a safety and mission success point of view. It was used to evaluate the predicted handling qualities, along with other design requirements. Therefore, it is necessary to evaluate the accuracy of these simulation predictions both qualitatively and quantitatively. Flight data can also be evaluated against the handling qualities metrics to evaluate the quality of the handling qualities predictions. The results discussed below highlight the comparisons between the simulation and flight from both an open- and closed-loop standpoint. All of the analyses presented use piloted frequency sweeps flown through the pilot stick and rudder pedals. The open-loop frequency responses are reconstructed from the closed-loop responses by identifying the individual components from Fig. 5 and recombining them in the frequency domain to form the open-loop frequency response. A method for determining frequency responses from flight data is presented in Ref. 15.

\section{Stability Margins and Open-Loop Frequency Responses}

Open-loop frequency responses and stability margins were used to tailor the NDI design. Therefore, comparisons between simulator predictions and actual flight-determined margins and responses provide valuable insight into the quality of the design tools and can be used to direct research to improve simulation models. The application of linear stability margins to a nonlinear control law in a nonlinear flight environment does not provide any guarantees of stability or robustness; however, it is common practice ${ }^{15}$ to evaluate fundamentally nonlinear problems in the linear regimen based on the assumption that the system behaves approximately linearly in a small region around equilibrium. Linear stability margins can be used to quantify the sensitivity of a nonlinear system to a class of linear uncertainties. Larger margins suggest that stability will likely be maintained in the presence of uncertainties that affect either the loop gain of the system or its phase.

Figure 6 shows a representative example of the pitch axis reconstructed open-loop frequency response of pitch rate error to filtered pitch rate. Since there are not significant differences between the results for the different FCs, and FC4 had the best overall coherence (not plotted), it is presented as the example open-loop pitch frequency response in Fig. 6. It is clear that the simulation predicted the flight characteristics very well, especially the phase response. This high-quality phase prediction suggests that the simulation accurately accounts for the primary sources of delay for the entire system. The most obvious discrepancy is the apparent offset in the magnitude plot in Fig. 6. It is clear that the flight pitch loop gain is nearly universally offset by a constant magnitude. The fact that this discrepancy is apparent in the magnitude of the loop and not in the phase suggests that the cause is either the pitch effectiveness of the pitch surfaces (likely symmetric stabilator) or arises from the fact that the commanded surface positions may not exactly match the actual resulting surface positions (unlikely, but not fully explored). In either case, the result is that slightly less pitch rate is being generated for a given command than was predicted by the 
simulation. In the simulation model, an overprediction of stabilator effectiveness of 10 to 20 percent resulted in responses that look very similar to Fig. 6. This result actually has a beneficial effect on most of the stability margins. As can be seen in Fig. 6 and Fig. 7, the reduced overall magnitude of the open-loop response shifts the gain crossover to the left (lower frequency) which results in a slight increase in phase margin. The fact that the phase plot in Fig. 6 is unaffected means that the phase angle crossover frequency is unchanged, and therefore higher gain margins result from the reduced magnitude plot. This gain shift also results in lower bandwidth for pilot control; the effects of this are discussed below. As seen in Fig. 7, the simulation predicts the overall margins and crossover frequencies very well; these pitch axis margins are consistent across the envelope shown in Fig. 4. At most of the FCs the controller has less than the desirable phase margin both in flight and in the simulation. One of the primary drivers for this deficiency is the additional time delay in the system that is inherent to experiments in the ARTS. Additional phase margin could have been realized by implementing less aggressive pitch reference models; however, this change would have had other undesirable effects from a handling qualities standpoint. Flight-validated phase margins of 40 deg or more are considered more than sufficient; therefore, it was decided that the reduction in bandwidth required to increase the phase margin was not necessary.
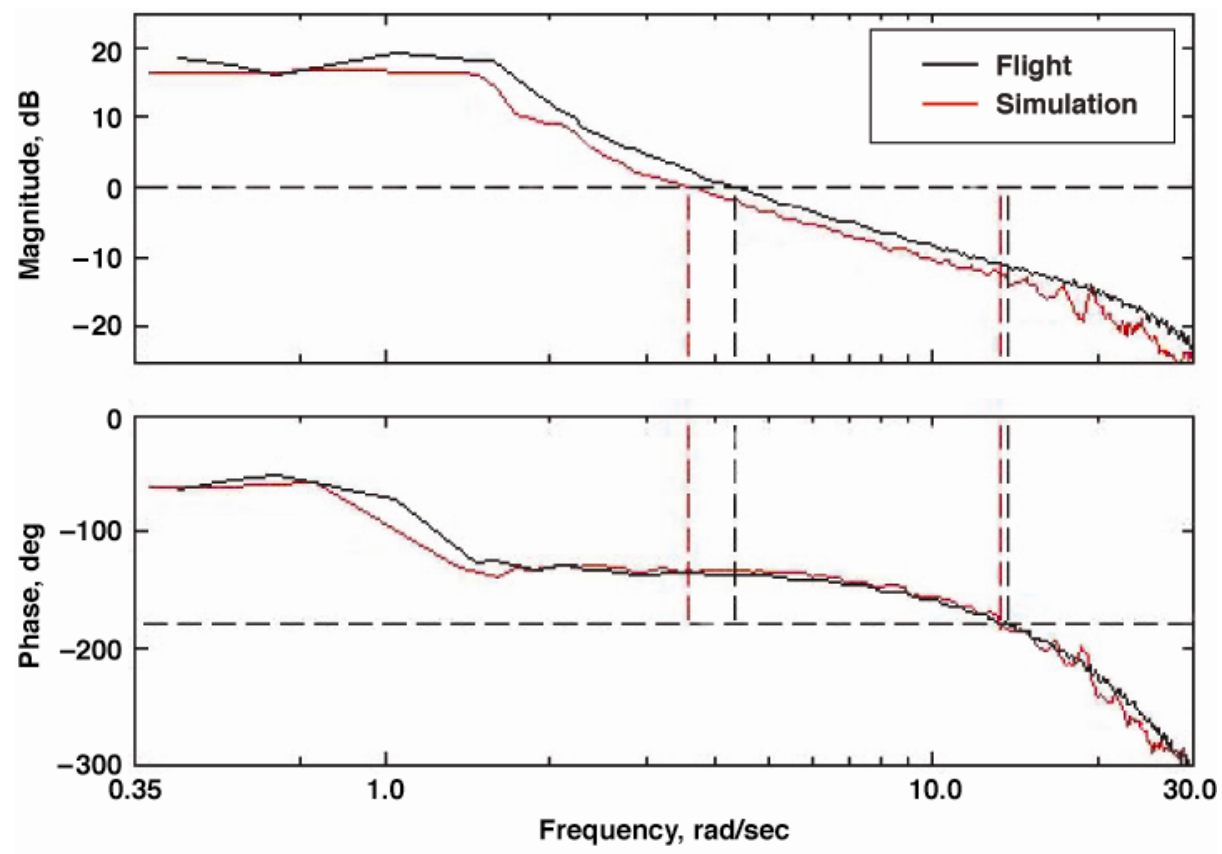

Figure 6. An example of the pitch open-loop frequency response comparison. 

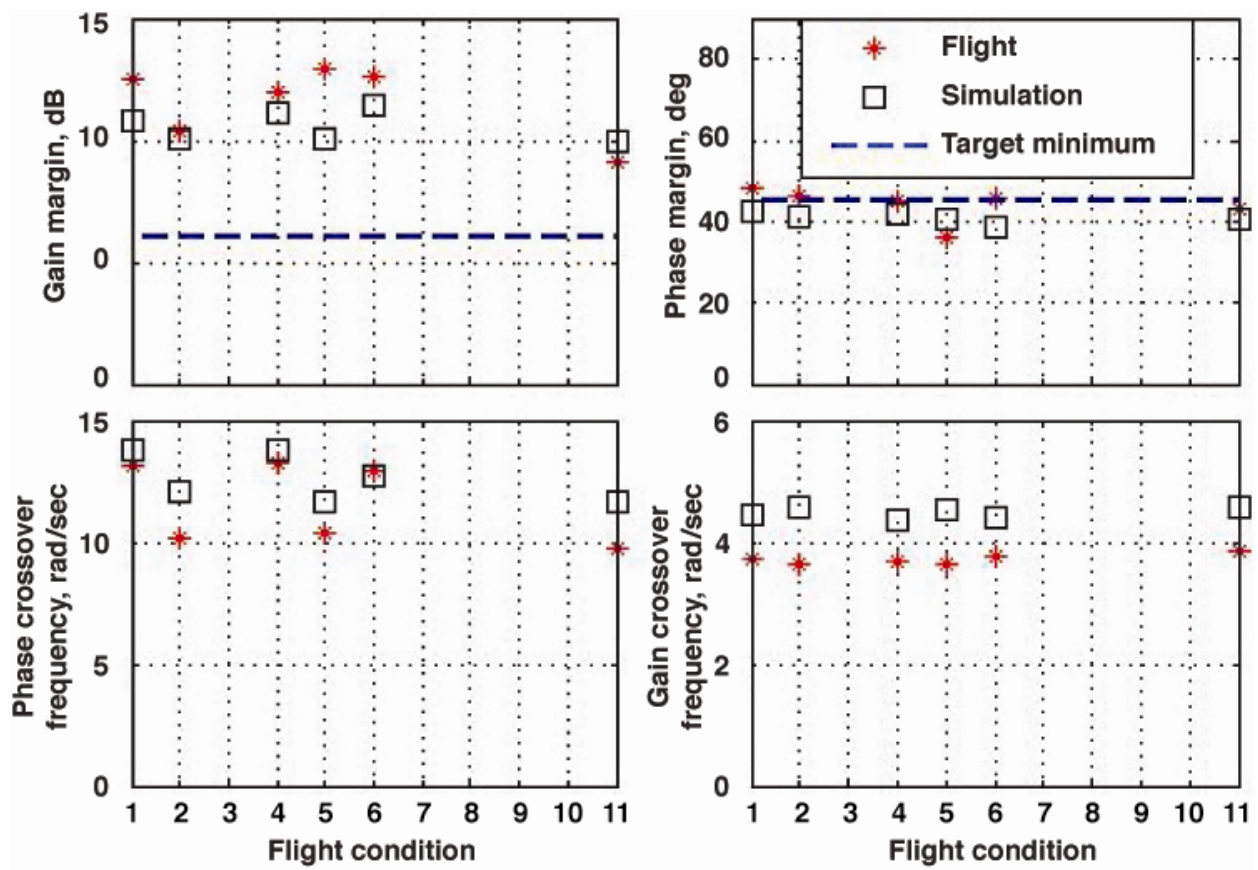

Figure 7. The pitch stability margins and frequencies for all flight conditions.

Flight-to-simulation gain and phase margin comparison in the lateral axis is even better than that of the pitch axis, as can be seen in Fig. 8 and Fig. 9. The simulation predicts the margins and their frequencies within the predicted precision of the numerical tools. As can be seen in Fig. 9, the roll axis has excellent stability margins that are consistent throughout the envelope given in Fig. 4. These results suggest that both the time delay and the surface effectiveness in the lateral axis are well-known and accounted for in the nonlinear hardware-in-the-loop simulation.
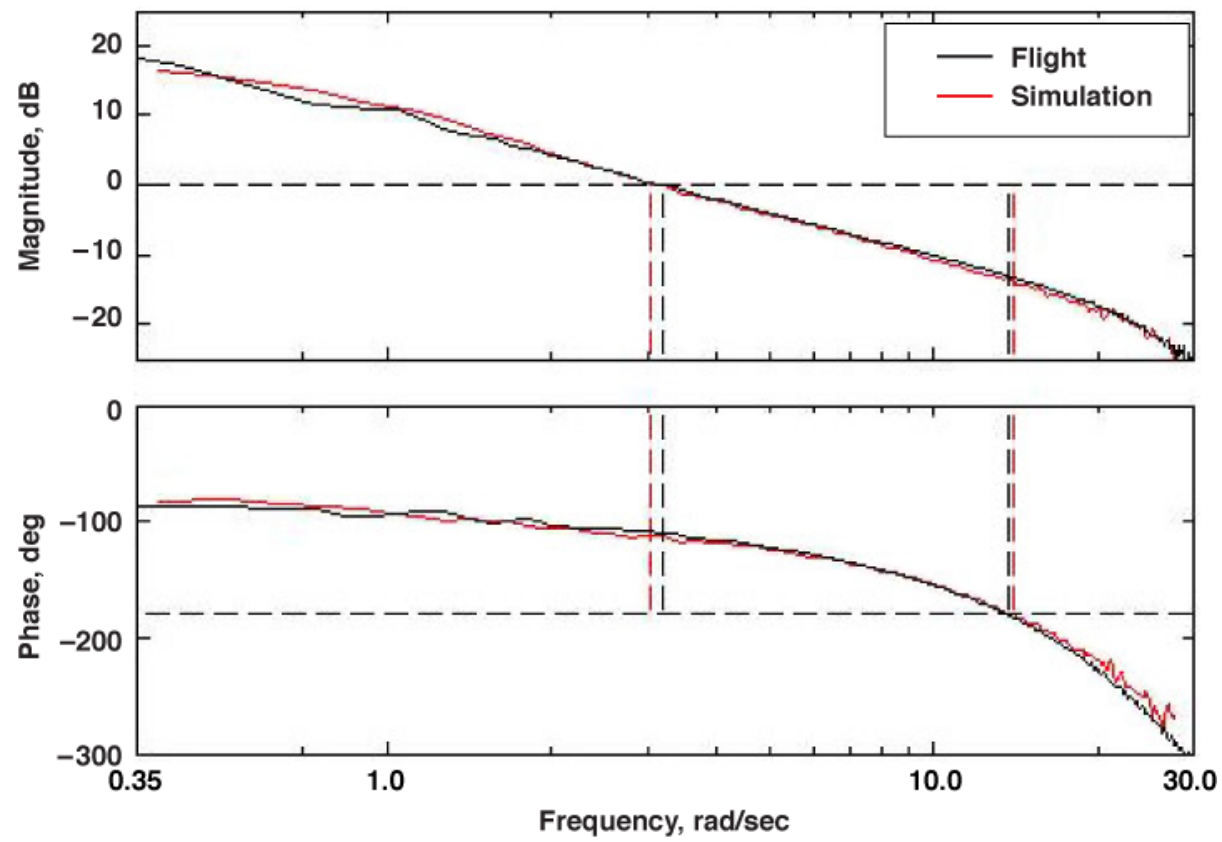

Figure 8. An example of the roll open-loop frequency response comparison (flight condition 4). 

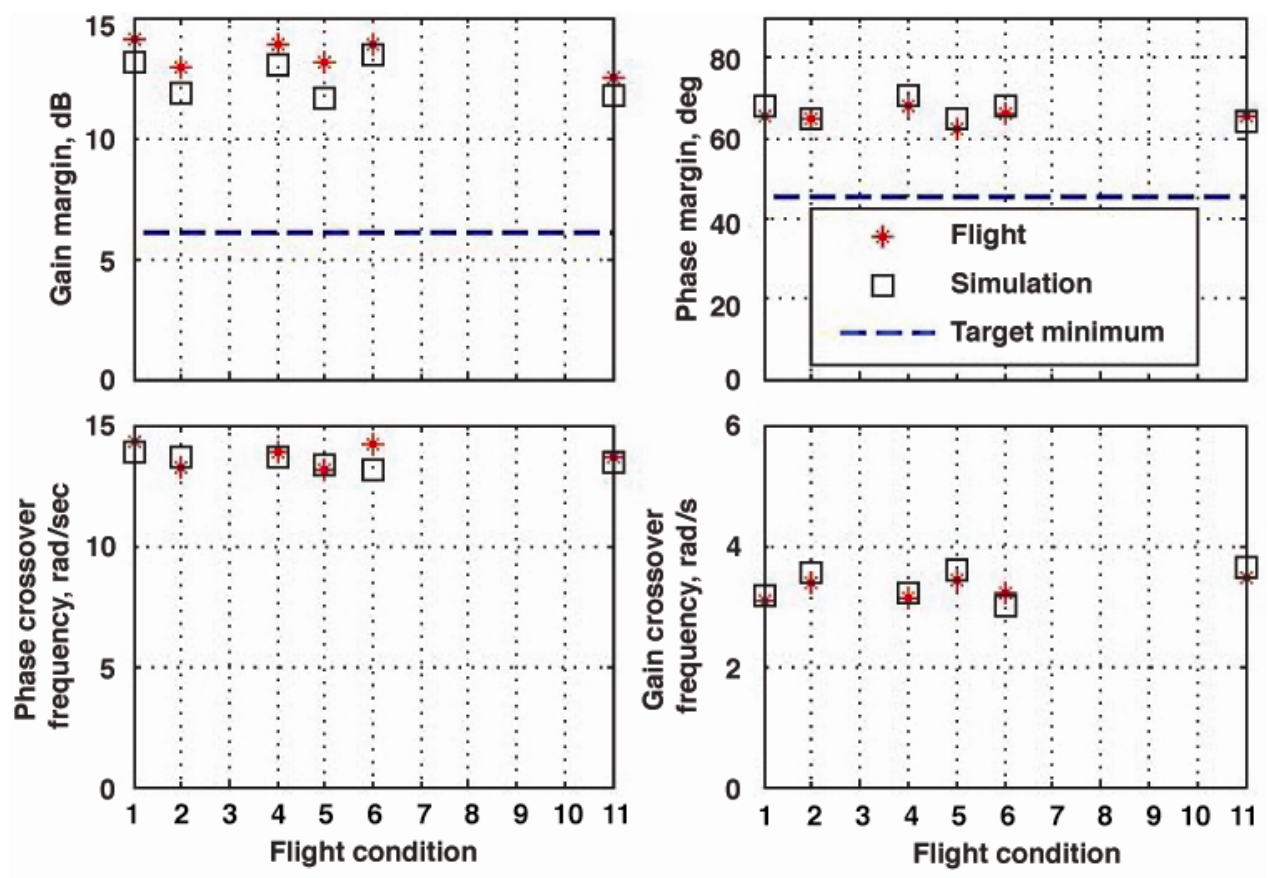

Figure 9. The roll stability margins and frequencies for all flight conditions.

The yaw axis shows a few interesting differences between the simulation and the flight responses. Figure 10 shows that the yaw axis behavior is fairly well captured, but there are some small differences that warrant exploration and explanation. The simulation prediction and flight results appear to diverge at approximately the $180^{\circ}$ crossover frequency. This divergence can be seen in both the gain and phase plots. The fact that the misrepresentation appears to affect both the phase and the magnitude suggests some unmodeled dynamics as opposed to a simple error in a model gain. This fact points to the onboard aerodynamic model as the likeliest source of the unmodeled dynamics because they contain the largest uncertainties. Specifically, it is believed to be attributed primarily to the homogenous (A-matrix) portion of the aerodynamic model. This assertion has not been fully explored because the effects of the modeling errors are small. It is also clear that the difference is not simply the result of some unmodeled source of time delay, because this would show up primarily in the phase plot. Other possible sources of error could include the sensor and actuator models in the simulation; however, there is currently no plan to explore the accuracy of these models. The results shown in Fig. 11 for the yaw axis stability margins are a direct result of the effects already discussed; however, it is clear that in the yaw axis very large stability margins exist both in the simulation and in flight. 

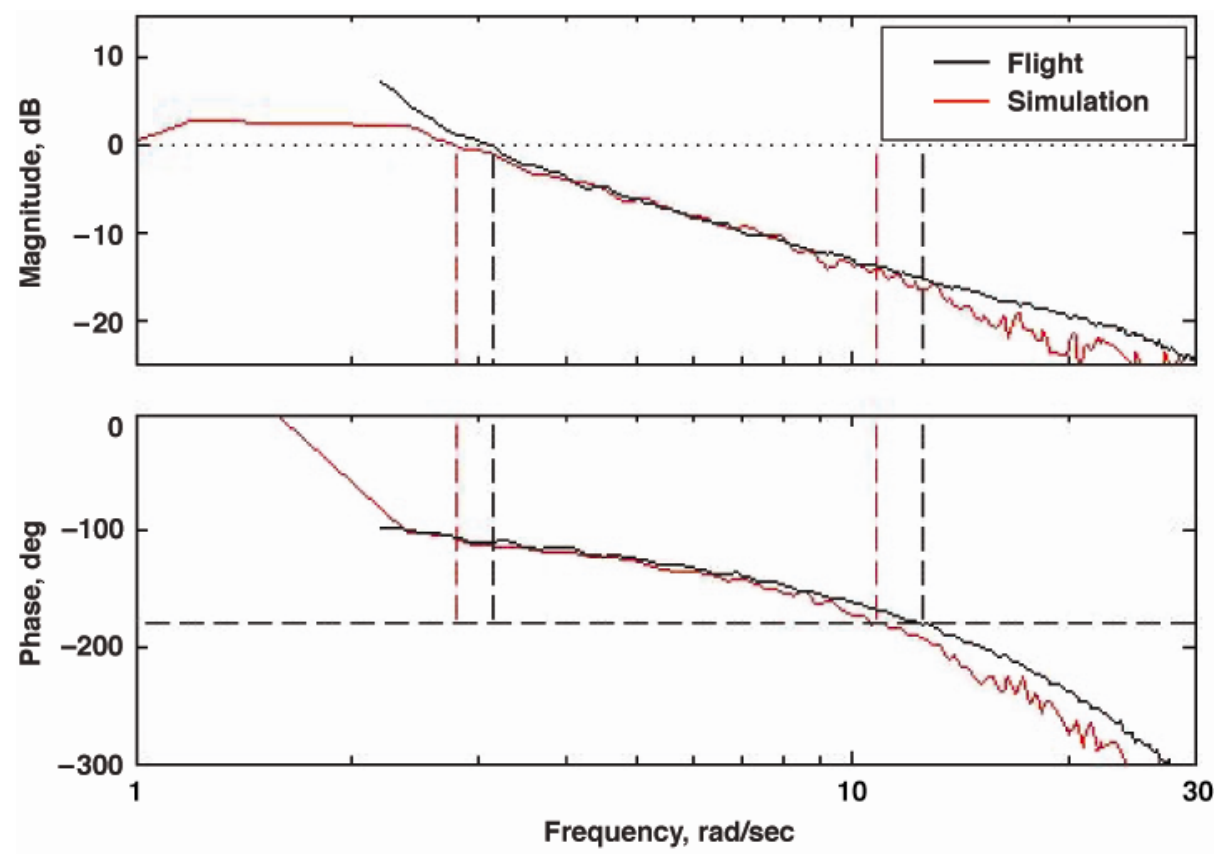

Figure 10. An example of the yaw open-loop frequency response comparison (flight condition 4).
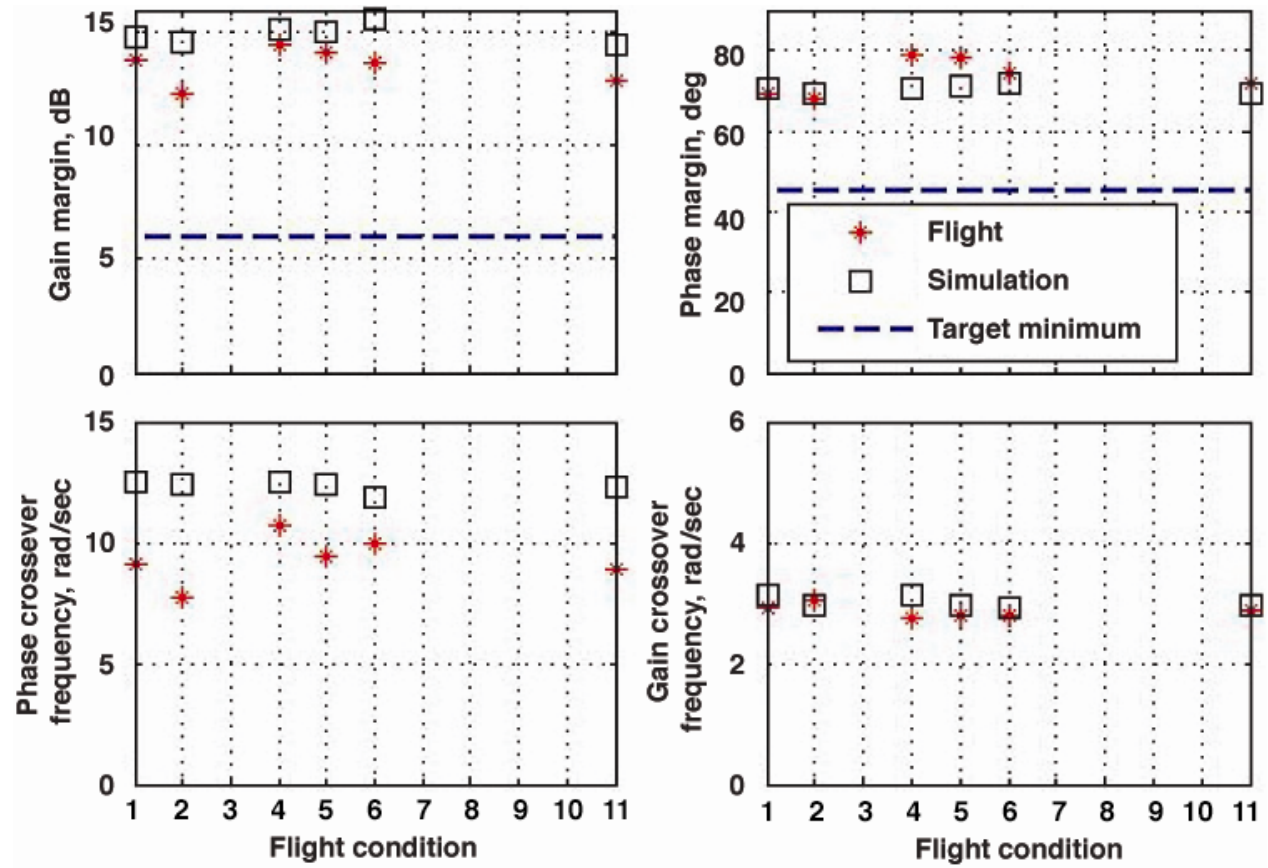

Figure 11. The roll stability margins and frequencies for all flight conditions.

These results show that from an open-loop frequency response and margins standpoint the NDI design outlined in Ref. 13 exceeds the design expectations, and that it does not suffer from any significant unmodeled or mismodeled dynamics problems. This result is important because it validates the tools and models used to design the NDI, and shows that both the facility and the baseline NDI control law are well-suited to explore advanced control law elements such as Model Reference Adaptive Control (MRAC). ${ }^{3,4}$ 


\section{Reference Model Tracking}

The choice of the reference model forms was driven by the existence of a wealth of experience in the handling qualities of the standard reference model forms from Ref. 16. It follows from this basic assumption that good tracking of reference models with desirable handling qualities ${ }^{16-20}$ should result in the closed-loop system exhibiting satisfactory behavior.

Good tracking, from a handling qualities perspective, is defined such that the pilot either cannot distinguish between the actual system and the design reference model or such that the pilot would give them equivalent ratings. ${ }^{21}$ Reference 21 establishes quantitative criteria for this property based on the pitch axis for a conventional aircraft with a center stick (pitch stick to pitch rate control systems). It labels the region in which the handling qualities of a dynamic system will be unchanged from a pilot perspective as the Maximum Unnoticeable Added Dynamic (MUAD) envelopes.

Figure 12 shows a good example of the flight-measured closed-loop tracking performance in the pitch axis. The desired tracking reference model in the pitch axis is the second order short-period approximation with time delay from Ref. 16. Only FC4 is shown because all of the closed-loop responses provided similar results, and FC4 had the most complete frequency content. Similar to the open-loop performance shown in Fig. 6, there is a small difference between the simulation prediction and the actual flight performance in the closed-loop gain at the moderate to high-frequency range. The integrator in the pitch axis proportional integral controller (PI) compensator eliminates the gain offset that was apparent in the open loop (see Fig. 6) in the lower frequency range. As stated previously, this magnitude difference most likely points to a small difference in the stabilator effectiveness. The primary effect of the reduced stabilator effectiveness is an apparent shift in the observed short-period natural frequency and a reduction in the resonant peak, which causes the magnitude plot in Fig. 12 to dip outside of the MUAD. The pilots commented that the pitch axis felt more sluggish with heavier stick forces than expected, which agrees very well with the quantitative results from Fig. 12. This apparent sluggishness can be fixed by simply retuning the pitch reference model short-period natural frequency, as discussed in Ref. 13. The sluggishness could also be fixed by using parameter identification to determine the actual stabilator effectiveness and then updating the simulation and the aerodynamic tables in the NDI. Since the error is small, that level of effort is unwarranted. The fact that the phase portion of Fig. 12 shows almost perfect agreement between the simulation and flight suggests that all of the important dynamics and time delays are modeled correctly in the simulation for the pitch axis.
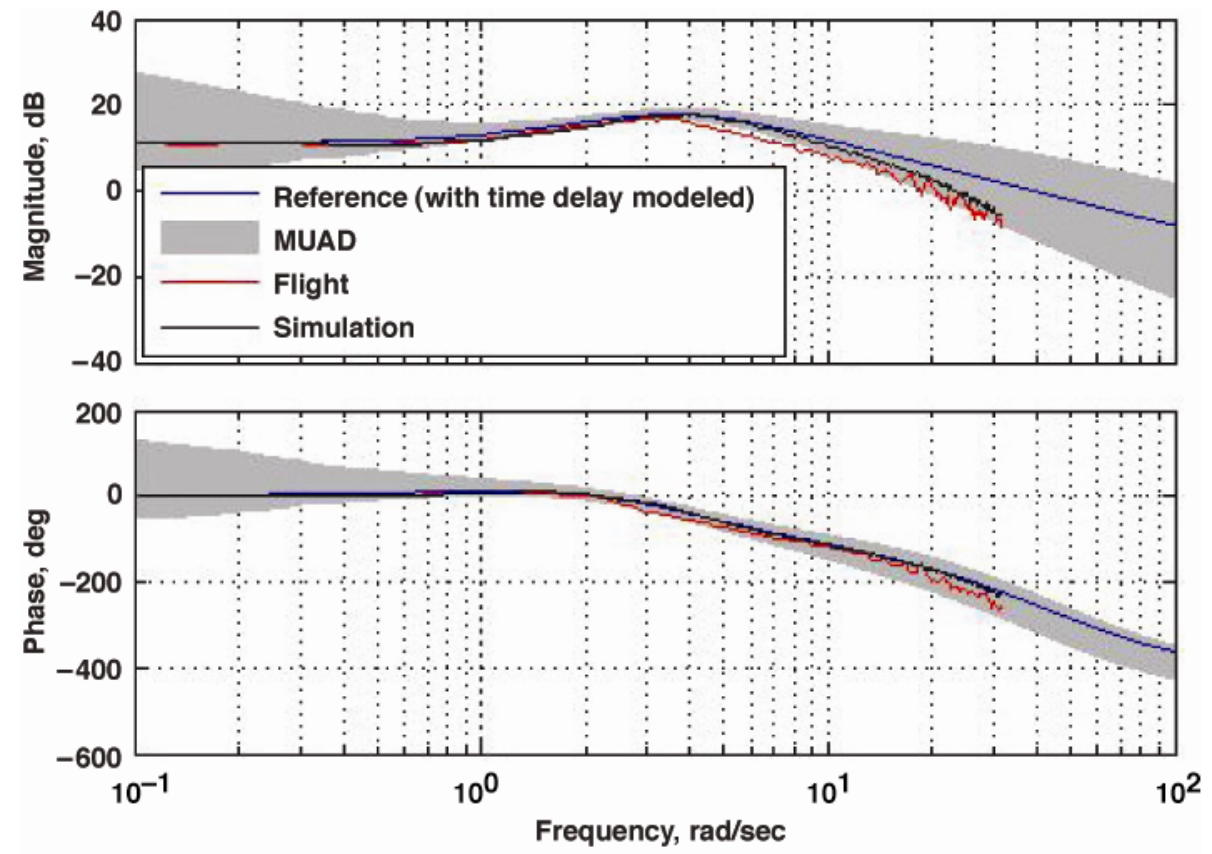

Figure 12. Closed-loop (pitch stick to pitch rate) pitch reference model tracking at flight condition 4.

The roll axis is better-behaved than the pitch axis. Figure 13 shows the tracking performance at FC4, which is representative of all the other FCs. The flight and simulation results are almost indistinguishable. The roll-off in the 
magnitude plot at the higher frequencies is related to actuator dynamics and is not a significant item from a handling qualities perspective. The MUAD is included on Fig. 13 simply for comparison purposes. It was not developed for the lateral axis, but it does provide some sense of how closely the actual flight response matches the desired response.
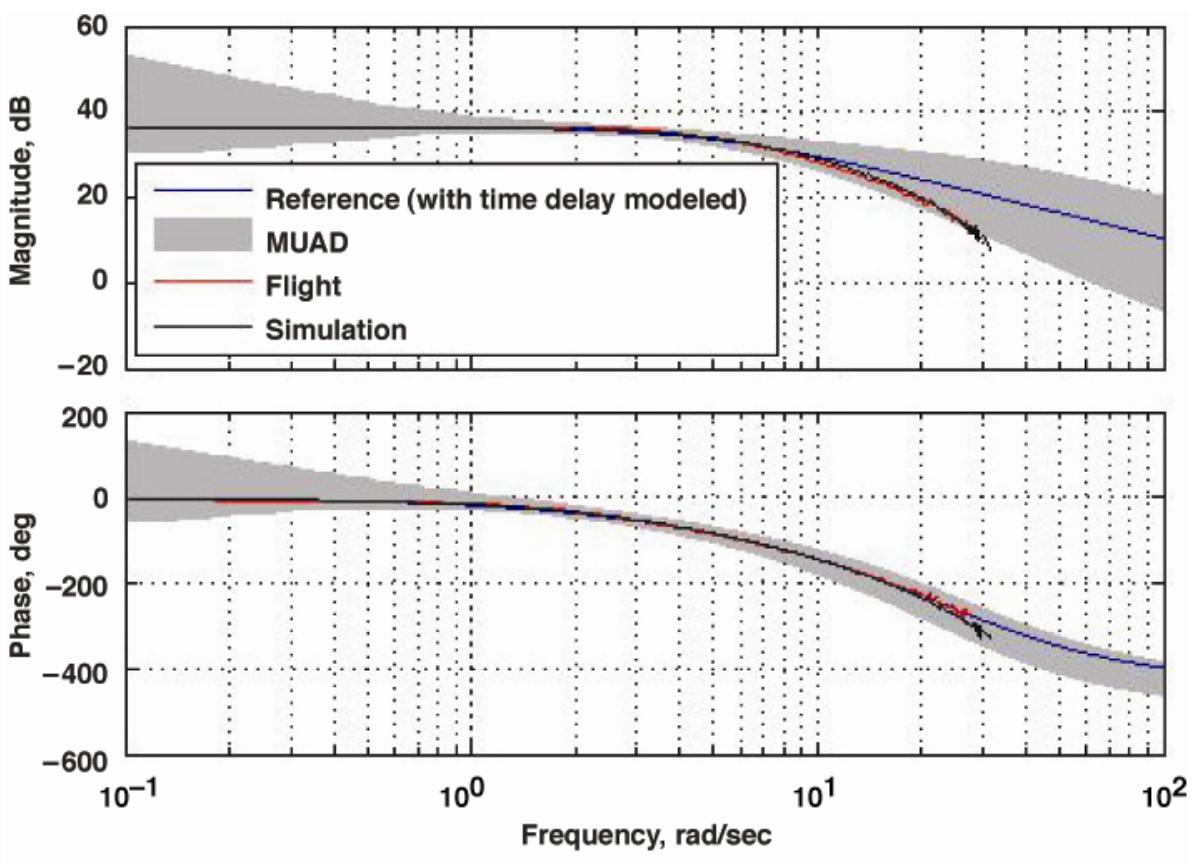

Figure 13. Closed-loop (roll stick to stability axis roll rate) roll reference model tracking at flight condition 4.

The tracking of the yaw reference model is not shown; it is poor in both the simulation predictions and the flight results due primarily to the lack of sideslip angle feedback. Sideslip angle, a fundamental quantity in the directional axis, is a state in the homogenous part of the equations of motion and has a major effect on the moments produced by the control surfaces. Currently, the dynamic inversion assumes zero sideslip, but better tracking of the yaw reference model could be realized with sideslip angle feedback. Feeding back sideslip angle comes, however, with other problems related to the reliability of the sideslip measurement. This additional complexity was found to be unnecessary; coordinated flight could be easily maintained even with poor yaw reference model tracking because of the stability properties of the directional axis of the bare airframe.

One additional comment on reference model tracking relates to managing larger envelopes. They are not presented here, but the tracking of the reference models at the lower impact pressure FCs (FC2, FC5, and FC11) was just as good as that at the higher dynamic pressure ones (FC1, FC4, and FC6); however, this tracking performance required significantly more aggressive actuator utilization at the lower dynamic pressures. This result suggests a strong advantage to gain scheduling the reference models with dynamic pressure, especially in the pitch axis. This scheduling was not necessary for the FCs (see Fig. 4) used for this research; however, as dynamic pressures decreased beyond those flown here, limit cycle oscillations in pitch and reduced stability margins were observed in the simulation environment due to actuator rate and position limiting. At higher dynamic pressure a pitch rate command architecture is prone to pilot induced oscillation (PIO) due to the rapid onset of g's for relatively low pitch rates. Elegant gain scheduling of the pitch reference model allows the normal acceleration response to be tailored even at high speed, and should prevent this PIO problem.

\section{Low Order Equivalent Systems}

Low order equivalent systems (LOES) analysis approximates a parameterized model from test data. The parameterized models can then be used to assess the overall system from a handling qualities standpoint. ${ }^{20}$ This technique is straightforward and a prevalent means for specifying longitudinal handling qualities requirements. ${ }^{16}$ The LOES technique is a natural way to evaluate the control law presented in this paper, as the dynamic inversion is designed in such a way as to track reference models of the exact form utilized by the LOES method. 
Figure 14 shows an example of a pitch axis LOES fit in the frequency domain when the frequency content of the input is very good. The frequency response data is smooth in the lower frequency range. High-frequency content inputs, however, are difficult for a pilot to generate. This limitation results in the jagged response seen at the higher frequencies in both Fig. 14 and Fig. 15. An automated test input used in place of the piloted frequency sweeps could have resulted in better results because it could have been tailored to have consistently good frequency content at all frequencies of interest. Figure 15 shows an example in which the frequency content is poorer. Getting good frequency content in the low frequency regime can be a challenge because large trim changes can result from them. In both figures it is clear, however, that the LOES model represents the overall behavior of the system well, and as a result provides a good basis from which to evaluate the handling qualities results.
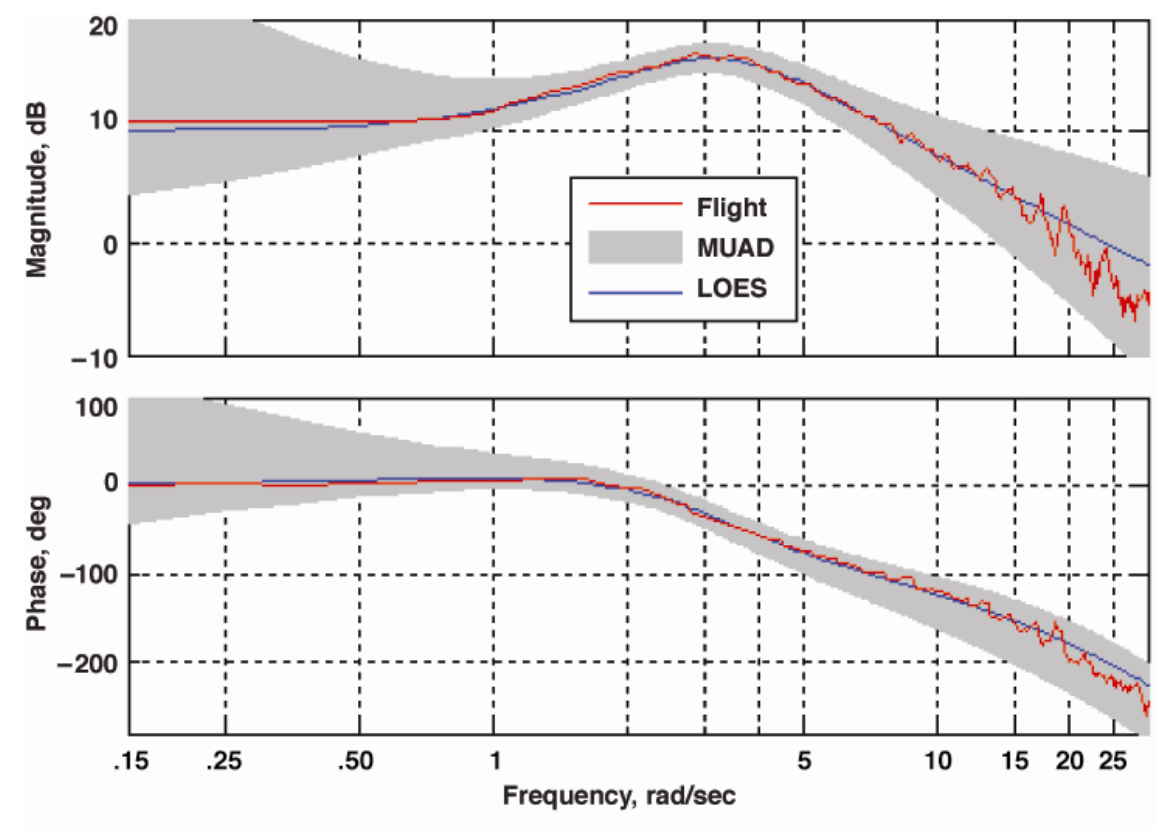

Figure 14. Pitch axis low order equivalent system at flight condition 4: high-quality frequency sweep.
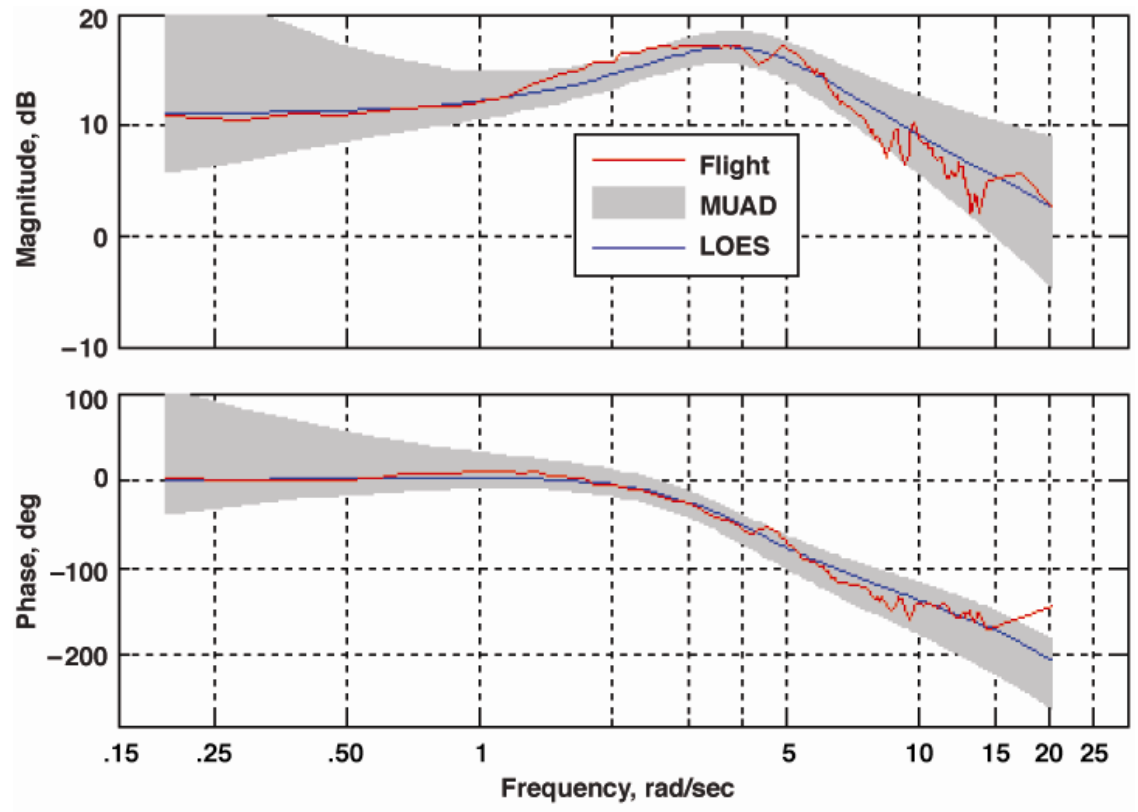

Figure 15. Pitch axis low order equivalent system at flight condition 11: low-quality frequency sweep. 
In the pitch axis only the short-period mode was considered in the LOES analysis. This decision stemmed from the best practices in Ref. 16. The first conclusion to note in Fig. 16 is that most of the values for the various parameters lie in the level 1 region. The control anticipation parameter (CAP) ${ }^{13,16}$ is not only level 1 , but the flight results closely match the predicted values and the design goal. The lower apparent natural frequency of the flight LOES when compared to the simulation predictions in Fig. 16 has been discussed above. This discrepancy has been attributed to a small discrepancy in the predicted effectiveness of the symmetric stabilator; however, it is clear that despite the fact that this effect is noticeable both in the LOES analysis and to the pilots it does not affect the overall handling qualities rating. The short-period damping is well-predicted by the simulation, but it can be seen in Fig. 16 that both are below the design goal of the reference model. This lower-than-desirable damping was found to be caused by the fact that actuator dynamics are not modeled or accounted for in the dynamic inversion in simulation studies. As discussed in Ref. 13, the actuator dynamics are empirically accounted for by detuning the reference models used to generate the actual commands to the NDI so that the closed loop matches the desired frequency response to a satisfactory extent. The equivalent time delay, which is really a measure of otherwise-unaccounted-for higher-order dynamics, can be seen to have less than desirable performance. It is well-predicted by the simulation; however, addressing the fact that it lies in the boundary between level 1 and level 2 is a complicated problem. Lag from sources such as actuators, sensors, structural filters, and the computational architecture are not easily addressed with a simple design change. Fortunately, the pilot comments (presented below) support the fact that despite not being ideal, the NDI time delay is acceptable and level 1 handling qualities are still attained.
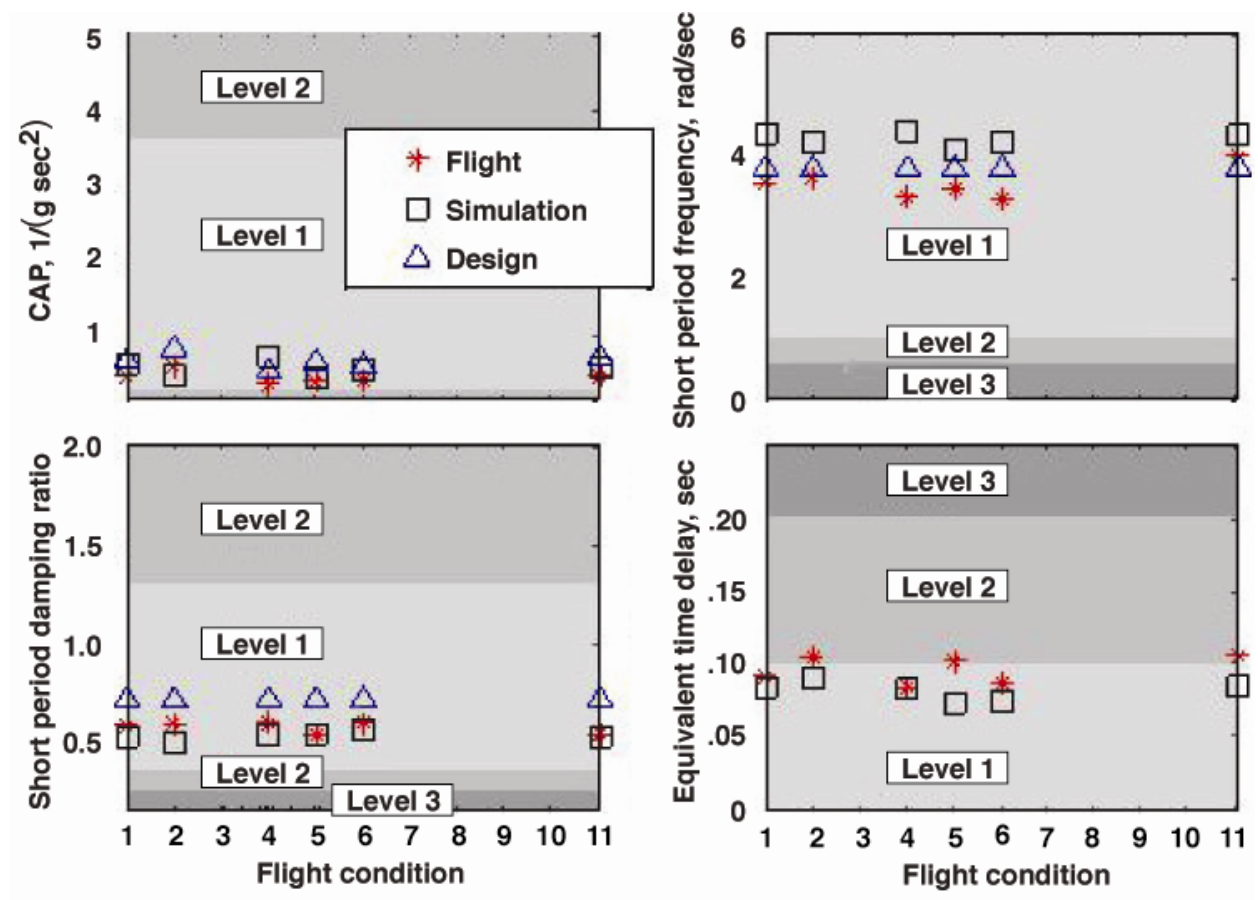

Figure 16. Pitch axis low order equivalent system results.

\section{Neal-Smith Criteria}

The Neal-Smith criteria is a mathematical-compensator- (simple-pilot-model-) based tool for quantitatively assessing the predicted pilot workload required to accomplish a pitch tracking task. ${ }^{18}$ The mathematical compensator in Fig. 17 is tuned such that a particular closed-loop bandwidth is achieved while minimizing the resonant peak in the closed-loop response. The lead compensation from this pilot model and the size of the resonant peak are then used to evaluate how difficult the task would likely be for a real pilot. Notable features of this analysis technique are that both the pilot model lag ( 0.3 seconds in Fig. 17), and the desired bandwidth are parameters that can be varied. Generally, 0.3 seconds and $3.5 \mathrm{rad} / \mathrm{sec}$ are used for the pilot lag and desired bandwidth, respectively, but variations in these parameters (particularly the bandwidth) can provide valuable insight into the sensitivity of the control law to aggressive piloting techniques. It is also desirable in some cases to use pilot lag other than 0.3 seconds, which was the case for the NDI. 


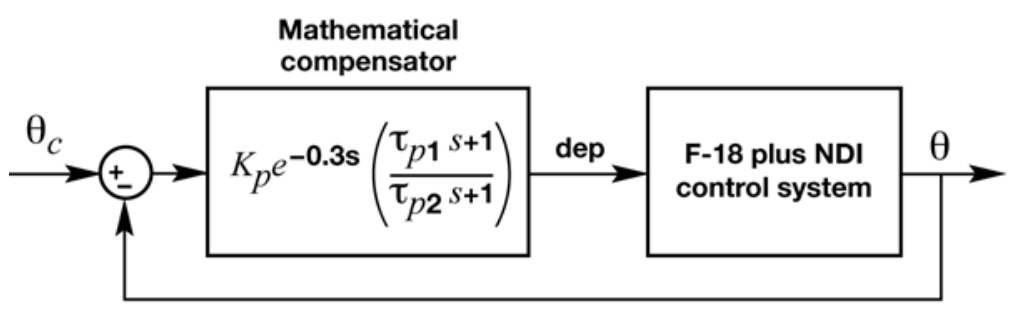

Figure 17. Neal-Smith compensator for the pitch tracking task.

Figure 18 shows how the NDI performs when evaluated with the Neal-Smith analysis method. The results in this figure were generated with two deviations from the standard Neal-Smith criteria. The first and most obvious is that, as previously discussed, a range of bandwidths were evaluated. Each of the traces in Fig. 18 contains nine different bandwidths. The point at the far left side is $1.5 \mathrm{rad} / \mathrm{sec}$. Each successive point increases by $0.5 \mathrm{rad} / \mathrm{sec}$ up to $5.5 \mathrm{rad} / \mathrm{sec}$. This range more than encompasses the range expected for pilots, but the gradual degradation of the predicted handling qualities with increasing bandwidth of each trace provides some confidence that the controller is not on the edge of having bad handling qualities. Another important difference is that instead of using 0.3 seconds for the pilot lag, 0.2 seconds was used. This decision was made based on the results of analyzing the production F/A-18 airplane control law (not shown). When the baseline F/A-18 airplane control law was evaluated with the standard Neal-Smith criteria, it was found that a pilot lag of 0.2 seconds matched the known level 1 characteristics of the control law better than the recommended value of 0.3 seconds. Therefore, 0.2 seconds was used to evaluate the NDI control law for all of the Neal-Smith analyses. The final modification to the technique was to use the LOES models generated in the previous section instead of the numerical frequency responses. The need for this modification can be seen in Fig. 15. The Neal-Smith analysis was found to be sensitive to the jagged nature of the numerical frequency response. The LOES model was used due to the fact that it was both smooth and found to model the closed-loop system very well.
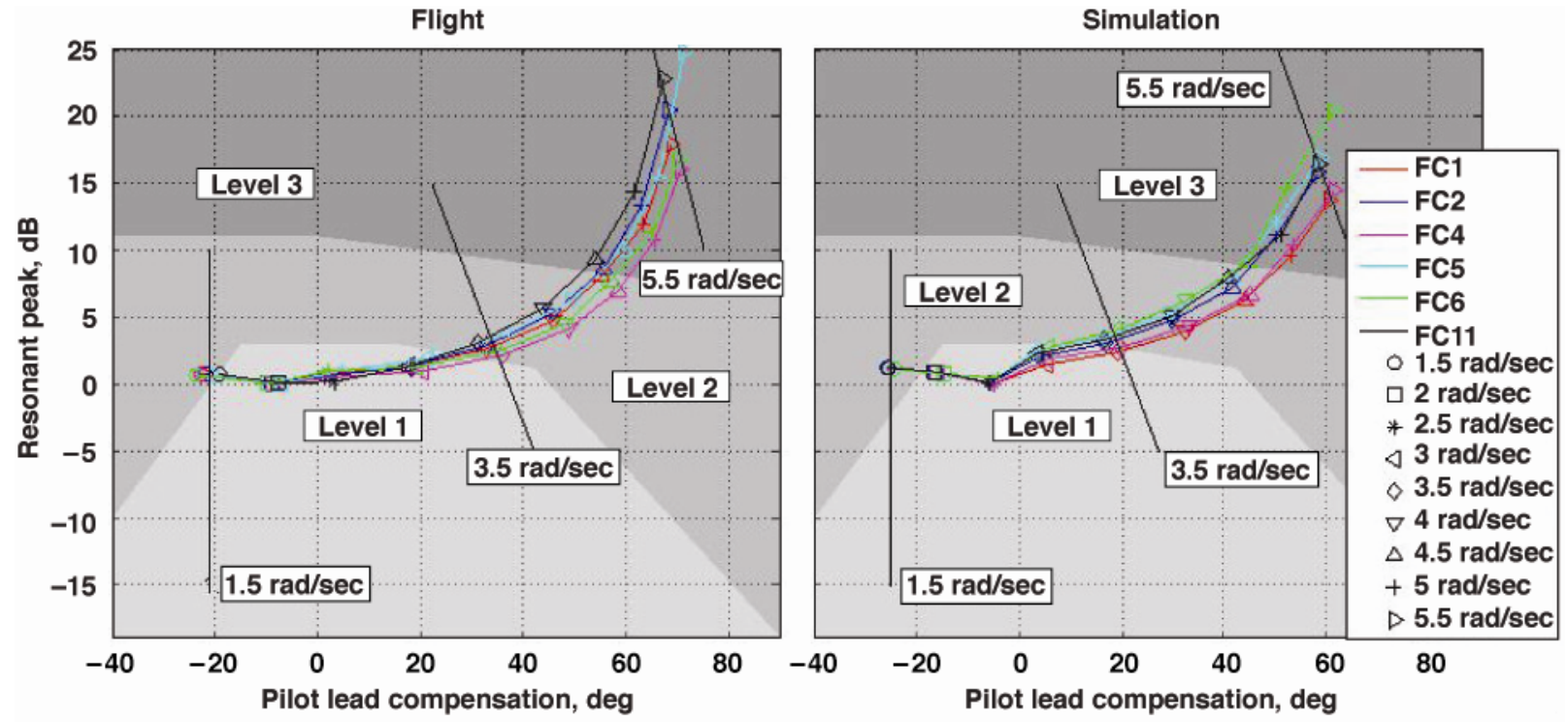

Figure 18. Pitch axis Neal-Smith results.

The flight data for the NDI controller that is shown in Fig. 18 suggests that the Neal-Smith resonant peak is more sensitive to increased bandwidth than was predicted by the simulation. This increased sensitivity can be seen more clearly in Fig. 19 in the increased slope of the lines drawn from the simulation results to the flight results. At about $3.5 \mathrm{rad} / \mathrm{sec}$ the slope changes from zero to positive, and the flight results show increasing resonant peak sensitivity with increasing bandwidth. This property contributed to a pitch bobble noted by all three pilots for aggressive pitch captures and by two of the three pilots for pitch tracking tasks (see the pilot comments discussion for more details). 
It can be seen in Fig. 18 that the lower dynamic pressure FCs (FC2, FC5, and FC11) suffered the largest handling qualities degradations in flight for increased bandwidth, which makes intuitive sense. At lower dynamic pressures the actuators have to move farther and faster due to reduced surface effectiveness; this behavior will limit the available bandwidth and cause larger overshoots for aggressive maneuvers. This dependency is not strong in the limited envelope from Fig. 4, but could play a more substantial role for larger envelopes suggesting the need for gain scheduling the reference models. Otherwise, the flight results confirmed the simulation-predicted handling qualities, and there are no notable required fixes or anomalies apparent from the Neal-Smith criteria.

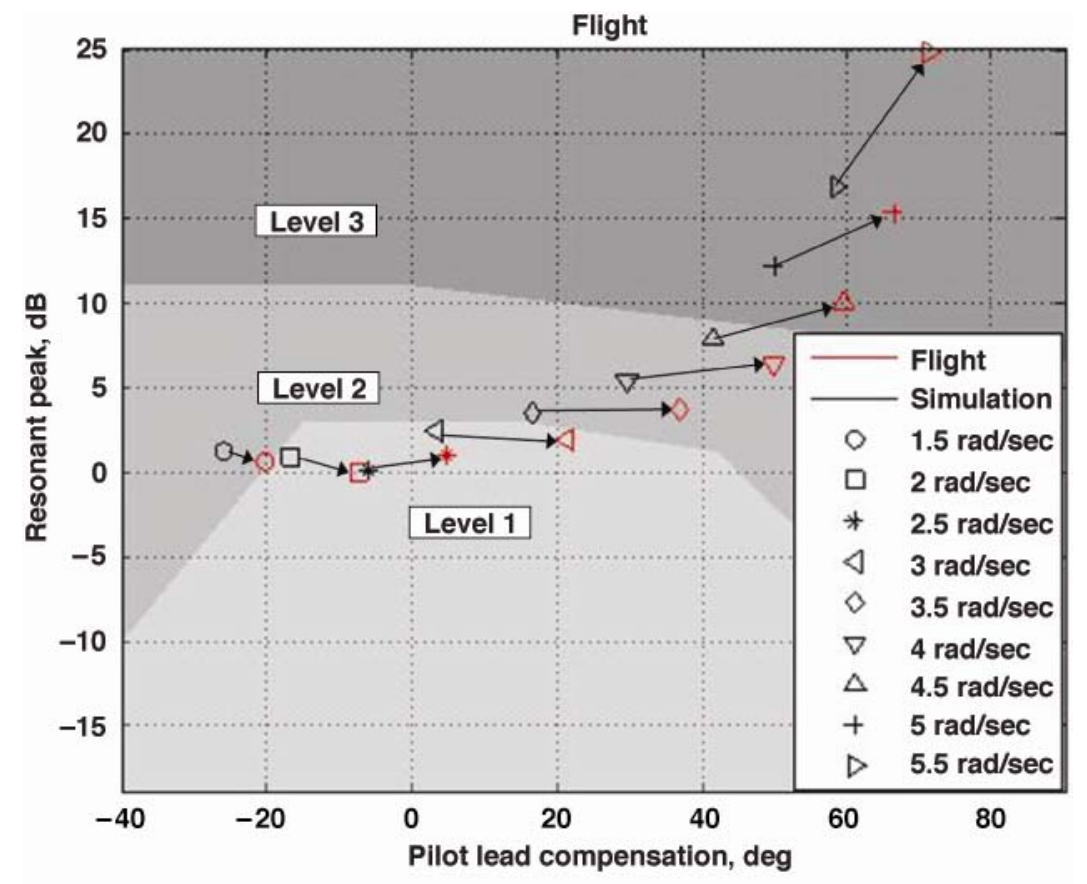

Figure 19. Pitch axis Neal-Smith results at flight condition 5.

\section{Bandwidth Criteria}

Many handling qualities metrics depend on the bandwidth of the total system. ${ }^{19}$ Reference 19 outlines a metric that is based on a specific definition of the bandwidth determined from the pitch frequency response of the vehicle and control system. One of the big advantages of this metric is that it also includes a prediction of PIO. ${ }^{17}$ Neither Ref. 17 nor Ref. 19 contain the most up-to-date handling qualities level boundaries when the feel system is included; the boundaries from Ref. 16 were used for the analysis presented below.

The bandwidth criteria use a specific definition of bandwidth and phase delay. See either Ref. 17 or Ref. 19 for a complete description of both of these important parameters. One last important parameter for the analysis presented in this paper is the prominence of the resonant peak of the pitch rate frequency response (see Fig. 12). If the magnitude of the peak is more than $9 \mathrm{~dB}$ above the lowest point to the left of it (normally the steady-state gain) a pitch bobble will likely be observed even in the level 1 region of Fig. 20. 


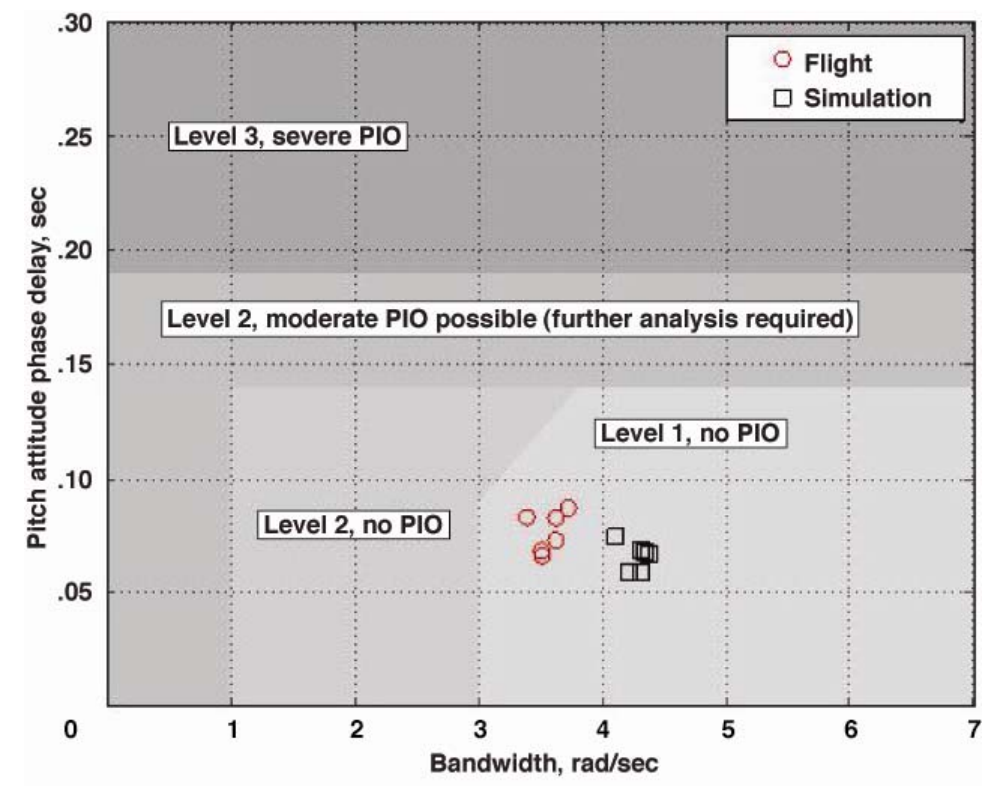

Figure 20. The pitch bandwidth criteria metric.

Figure 20 and Fig. 21 show that in general the flight results show an obvious reduction in control bandwidth pushing the handling qualities closer to the level 2 boundary; however, level 1 handling qualities are maintained. This reduction in bandwidth is likely a direct result of the fact that the pitch surface effectiveness (primarily stabilators) appears to be less than was predicted by the simulation aerodynamic data (discussed above). The apparent reduction in bandwidth can be addressed by tuning the pitch reference model based on this flight result. In addition to the bandwidth reduction, a small but noticeable increase can be seen in the pitch attitude phase delay. This increase is small enough that it was not apparent in the other analysis techniques. The simulation models all the known sources of delay, therefore, the increase in phase delay is likely the result of either some additional lag in the actuators, or a sensor; however, it may be simply an artifact of the data recording system. It is clear, however, that this small increase in pitch attitude phase lag is not significant from a handling qualities perspective. Overall, the simulation provided high-quality predictions, and the metric performs well on the flight data from the NDI.
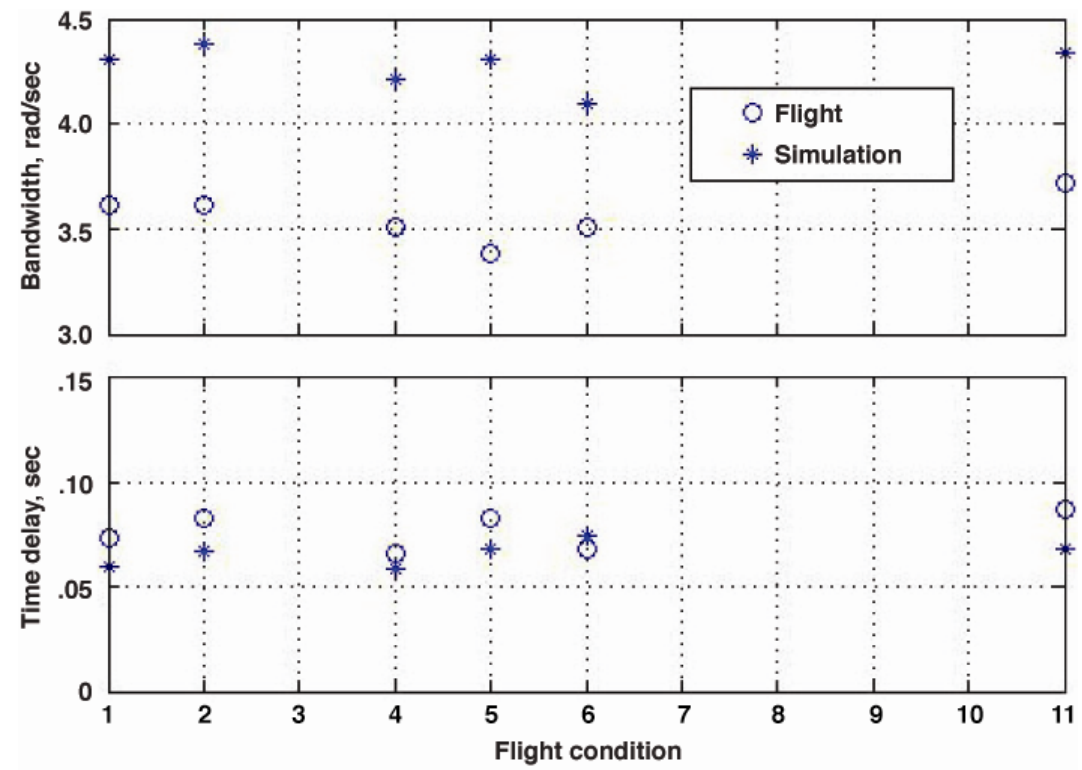

Figure 21. Pitch bandwidth criteria versus flight condition.

17

American Institute of Aeronautics and Astronautics 


\section{B. Pilot Comments and Ratings}

Pilot opinion can make or break any new control law design for a piloted vehicle. Therefore, understanding the meaning of pilot comments, determining what pilots consider desirable flying characteristics for an array of tasks, and understanding what cues and inputs they are using to accomplish those tasks are essential to achieving desirable handling qualities. The NDI was evaluated by three different pilots for a number of basic maneuvers and two closed-loop tracking tasks. All three pilots are experienced test pilots with significant experience flying unique configurations. Pilot A has substantial glider experience, flew reconnaissance aircraft operationally, was an instructor pilot in various aircraft, and instructed at the United States Naval Test Pilot School. Pilot B has significant experience flying both light and heavy fighter aircraft and business jets. The bulk of Pilot C's flight hours are in high-performance fighter-type aircraft; however, as a 30-year test pilot he has flown a large variety of aircraft including bombers, cargo aircraft, and business jets.

The concept of pilot gain is a useful abstraction when considering how aggressively a pilot approaches a given task. In a sense, it reduces the pilot to a linear proportional feedback controller and allows the engineer to qualitatively differentiate between different piloting techniques. This approach is overly simplistic, but it provides a useful way to describe the complex subject of piloting technique. In the following discussions pilot gain is used to describe how aggressively a given maneuver was performed. Reference 22 provides more detail about this subject and discusses some of the limitations of this type of approach.

\section{Basic Flying Qualities}

The basic flying qualities of the NDI were assessed with the following maneuvers: doublets in each axis, pitch and bank captures, steady-heading sideslips to maximum rudder pedal, $360^{\circ}$ rolls with half of the maximum lateral stick, 2.5-g wind-up turns, and 2-g loaded rolls with half of the maximum lateral stick. These basic maneuvers were used to determine the characteristics of the control law prior to the tracking and formation tasks. All of the pilots flew the basic maneuvers with the NDI at FC6 (see Fig. 4) and each pilot flew at least one other FC clearing the entire envelope shown in Fig. 4. Flight condition 6 was the primary focus, however, because this was the flight condition in which the handling qualities tasks were to be performed both for this experiment and the follow-on MRAC experiment presented in Refs. 3 and 4. In general, the pilots' comments compared the NDI characteristics to the production F/A-18 airplane control law.

Pilot A flew the NDI at FC6, FC5, and FC4, in that order. At FC6 he commented on a mild pitch bobble tendency during the pitch captures that was less pronounced than in the simulation but still apparent and felt it was likely to affect pitch tracking tasks. He felt that the pitch predictability was still good, however, and noted that that the bobble dies out quickly, producing one or two overshoots. This bobble is the result of the magnitude of the resonant peak in the pitch magnitude frequency response being too high above the steady state (see Fig. 12), and could be addressed by a change in the pitch reference model. He found the bank captures to be easy, but that the NDI tended to halt the roll more crisply than the production control law, which tended to cause undershoots of the desired bank angle. He was able to compensate easily and found the differences to be unremarkable. His primary negative comment for the NDI was in reference to the pitch response during $360^{\circ}$ half-stick rolls. He noted that the pitch response tended to result in him being light in the seat while inverted. All three pilots had the same negative impression of this behavior, and pilot A noted a tendency for this problem to be exacerbated by slower roll rate $360^{\circ}$ rolls. This undesirable feature of the control law is a result of the pure pitch rate feedback architecture; a simple fix is suggested in Ref. 13. At FC4 and FC5 pilot A did not note any significant differences for the NDI compared to the behavior at FC6. He commented, however, that at FC5 the roll and pitch responses were both faster than would be expected for a standard F/A-18 airplane at those slow calibrated airspeeds. This difference resulted in him having to compensate by leading the airplane less during the bank captures than expected, which ended up being desirable after performing the task a couple of times.

Pilot B flew the NDI at FC6 and FC1. He commented that at both FCs it was unremarkable and that it flew like the simulation predicted, including the pitch bobble during the pitch captures. As noted previously, he found the pitch response during inverted flight to be undesirable.

Pilot C, who flew the NDI at FC6, FC2, and FC11, had comments similar to those of the other two pilots, with some noteworthy additions. He noted a hesitation in pitch during the pitch captures at FC6. This comment points to the fact that the pitch bobble may be related to the frequency at which the resonant peak in Fig. 12 occurs, rather than the bobble being caused solely by the magnitude of the resonant peak. It was noted in the "Low Order Equivalent Systems" section above that the flight-measured frequency response did exhibit a lower short-period natural frequency and this may be adding to this pitch bobble tendency. This shift in frequency can be addressed with a simple reference model change. Pilot $\mathrm{C}$ commented that the roll response was very precise at all FCs. He commented that the NDI was very responsive in roll at FC2 and exhibited desirable traits especially at such a slow 
calibrated airspeed, which is similar to pilot A's comment at FC5 (same calibrated airspeed). However, he found the unload that was exhibited during the $360^{\circ}$ rolls at all FCs to be particularly objectionable, which again is similar to the other two pilots. He did not have any unique comments about the control law at FC11, which is as expected.

The last item to note regarding the pilot comments from the basic maneuvers is related to the speed brake. No compensation for speed brake effects was included in the NDI formulation, which results in a small pitch-up transient when the speed brake is deployed. None of the pilots found this transient to be objectionable; they commented that the transient was similar to the production control law, which does include speed brake compensation. The PI compensator successfully keeps this transient small as expected and desired.

\section{In-Trail Formation Task}

The in-trail formation task, which was flown only at FC6, is intended to be representative of an en-route formation flight task. This task requires the pilot to maneuver the vehicle in both the longitudinal and lateral axes simultaneously. This property makes it a good task for evaluating control harmony between axes and it highlights any undesirable coupling of the axes. Reference 22 suggests that a similar task is useful for predicting lateral-directional tracking characteristics for offset landings. Figure 22 and Fig. 23 illustrate the in-trail formation maneuver. The test aircraft starts directly behind and below the lead aircraft. The pilot then maneuvers the aircraft aggressively outboard and up and attempts to track the missile rail of the lead aircraft. Refer to Fig. 23 for the visual references used for tracking and maneuver setup. Unfortunately, the nature of the task and the available piloting cues precluded the use of anything except pilot opinion for evaluation of his performance at meeting the desired and adequate criteria. These criteria are presented in table 2. Additionally, this task is more sensitive to the longitudinal separation of the two vehicles than desired due to the wake of the lead aircraft. This undesirable aspect of the task forced an increase from one ship length of separation for the results presented in table 3 to two ship lengths of separation for the MRAC tests. ${ }^{3,4}$ This increase in separation helped with repeatability; however, it changed some aspects of the task such as pilot aggressiveness effects. Additionally, it would have been more desirable to perform a tight formation task on the wing of the lead aircraft; however, the limited experience with the ARTS IV architecture and the limited verification and validation testing performed on the NDI control law precluded maneuvers during which the vehicles overlap longitudinally. Future experiments will not necessarily have this same restriction, depending on the level of verification testing.

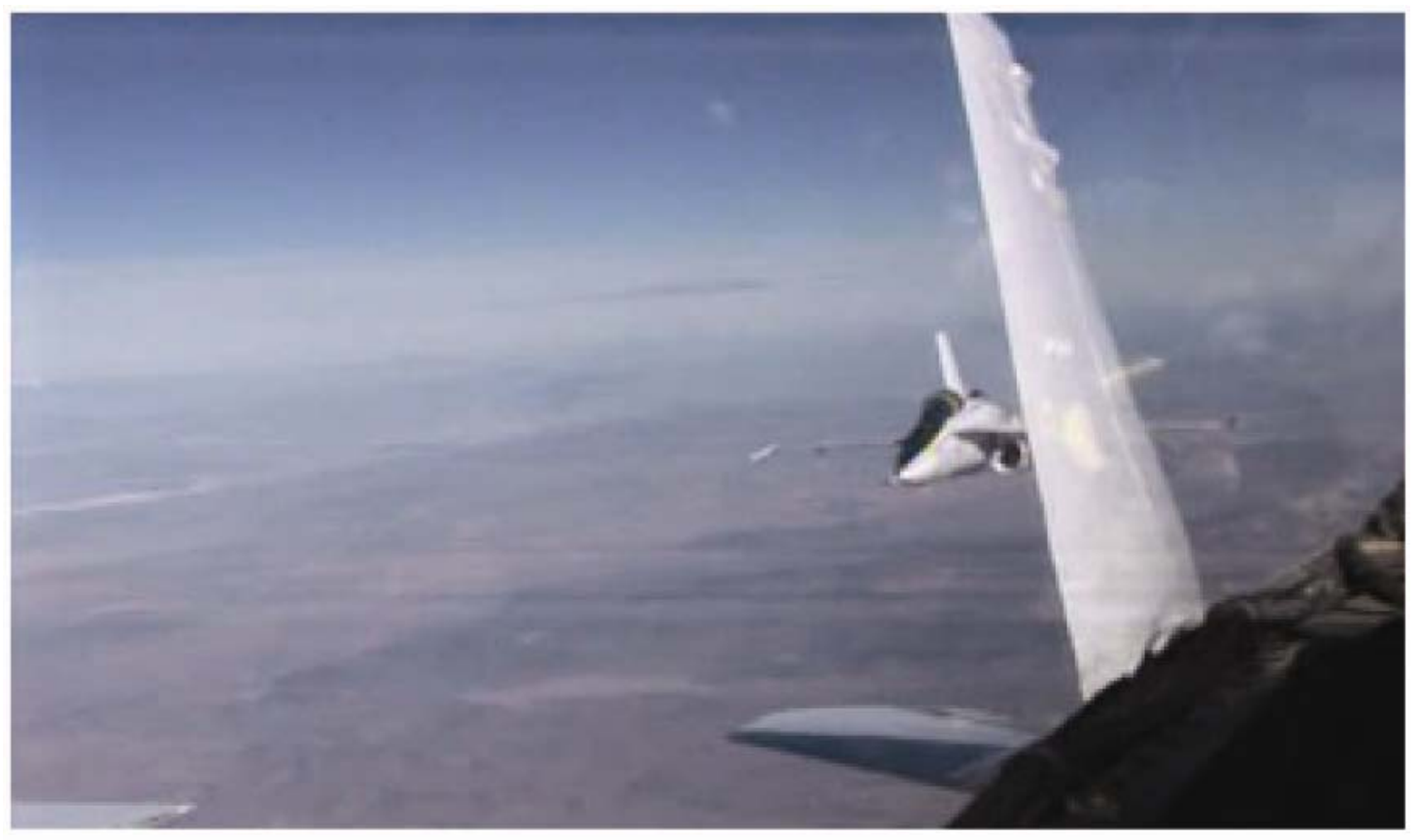

Figure 22. Lead aircraft view of the text aircraft during the fine tracking portion of the in-trail formation task. 


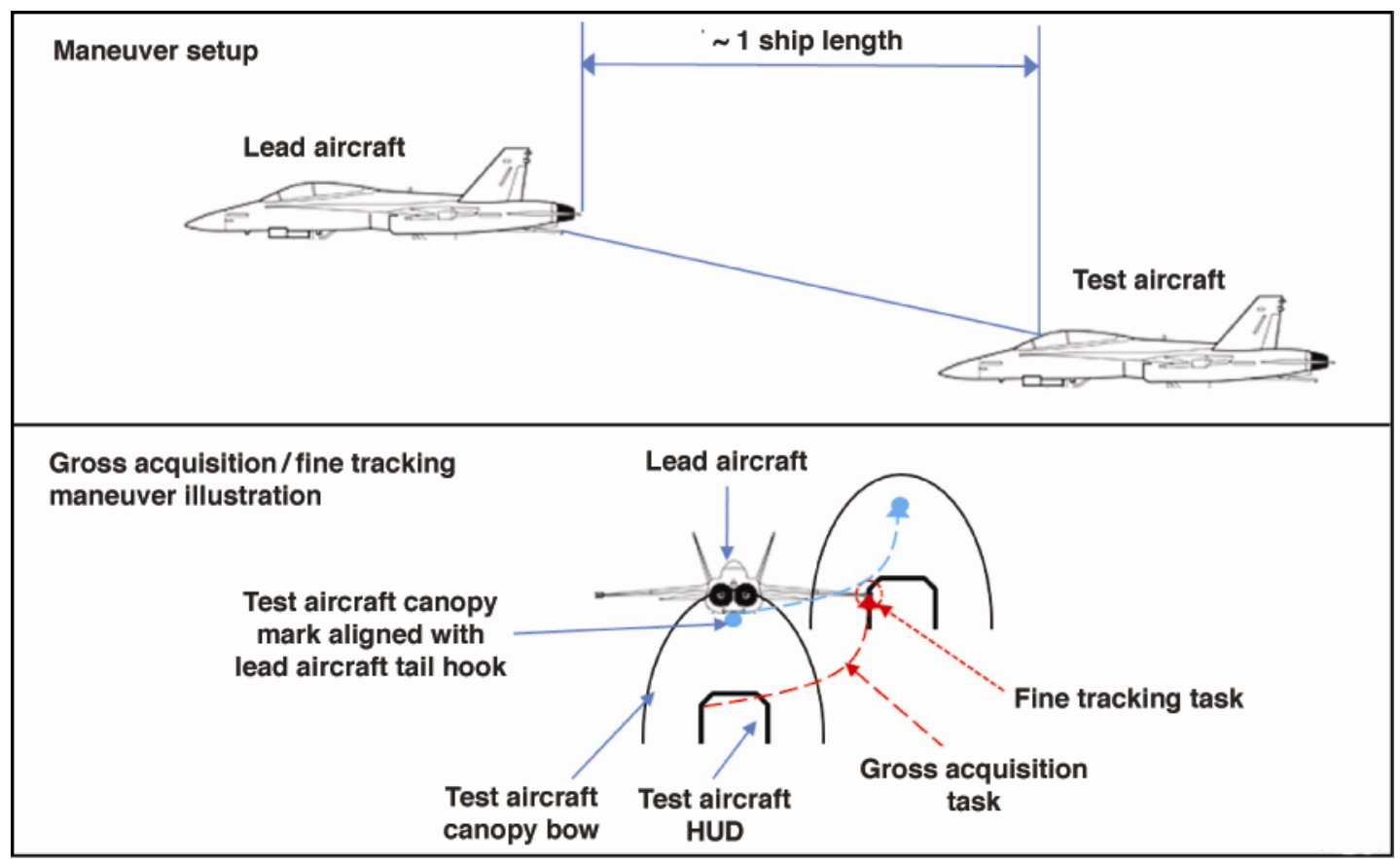

Figure 23. Illustration of the in-trail formation task.

Table 2. Performance criteria for the in-trail formation task.

\begin{tabular}{lll}
\hline \hline & Gross acquisition & Fine tracking \\
\hline Desired & $\leq 1$ overshoot & Bracket on missile rail $80 \%$ of the time \\
Adequate & $\leq 2$ overshoots & Bracket on missile rail $50 \%$ of the time \\
\hline \hline
\end{tabular}

Table 3. Cooper-Harper pilot ratings for the in-trail formation task (ratings prior to practicing the task).

\begin{tabular}{lcccc}
\hline \hline & \multicolumn{2}{c}{ Gross acquisition } & \multicolumn{2}{c}{ Fine tracking } \\
\hline & Production & NDI & Production & NDI \\
Pilot A & 3 & 3 & 4 & 4 \\
Pilot B & 4 & 5 & 2 & 4 \\
Pilot C & 2 & 4 & 2 & 3 \\
\hline \hline
\end{tabular}

Table 3 shows the Cooper-Harper ratings ${ }^{23}$ that each pilot gave the production control law and the NDI for the in-trail formation task. It is noteworthy that these ratings were not collected in a blind fashion, and that the pilots were aware of the configuration they were testing along with its predicted characteristics. Additionally, training on the task in the production mode was performed before any tests for score were executed. These factors may have affected the ratings; however, due to the abbreviated schedule, the decision was made to perform pilot familiarization and control law checkout in parallel with these test maneuvers. The decision to perform the maneuvers in a predefined order was made by the project team in order to ensure the safest possible flight program within the shortest possible schedule.

Pilot A commented that the differences between the production control law and the NDI were nearly imperceptible, with the NDI exhibiting less undesirable roll motions. Pilot A's ratings are supportive of this assertion. He commented that the control harmony and forces were desirable for both, and that both controllers tended to produce a bounded roll oscillation (possibly a PIO) for the gross acquisition task when he was very aggressive. He compensated for this by consciously backing out of the loop slightly. Pilot A was asked to repeat this task with the NDI three months later at the conclusion of the MRAC flights, ${ }^{3,4}$ with a longitudinal separation of two ship lengths, and his ratings improved by one for both the gross acquisition (GA) and fine tracking (FT) tasks 
(GA 2, FT 3). His comments about the task changed slightly as well. He commented that there were no undesirable motions where previously he noted a bounded roll oscillation that increased in magnitude with increased aggression. He still commented, however, that increased aggressiveness did negatively affect his performance. This apparent change in pilot opinion was primarily a function of the increased vehicle separation and the effect it had on his aggressiveness. Another aspect of the test that had an effect on the pilot opinion was that the pilot was learning the task and the characteristics of the NDI control law throughout the testing. He was able to achieve desirable performance with acceptable levels of compensation for all tasks performed.

Pilot $B$ and pilot $C$ noted some degradation in the NDI as compared to the production control law, as can be seen in table 3. For pilot B, unfortunately, the quality of the audio recording was poor, so some of his comments were not captured. He commented about the roll response being predictable but slower than the production vehicle, and that he experienced some undesirable yaw motions. When he repeated the task at the end of the MRAC flights with the larger vehicle separation, his impression of the task and the control law changed and he did not note any undesirable motions or comment on the slowness. His ratings changed to 3 for GA and to 2 for FT for the NDI. The larger separation also affected his aggressiveness, which masked some of the shortcomings of the NDI related to pilot gain.

Pilot C's comments highlight some substantial differences between the NDI and the production control law. He felt that the forces in roll were heavy for the NDI and that it caused the response to be sluggish, requiring him to compensate by leading the airplane more for the GA task. This description of the behavior of the airplane supports pilot A's comments about aggressiveness causing a bounded roll PIO, and agrees with pilot B's comments about the slowness of the NDI for this task; the slowness is causing the pilots to over-control the airplane laterally for this task. It also suggests that the stick shaping in the roll axis discussed in Ref. 13 may be too aggressive for this task. Other possible sources of this sluggishness are the roll mode time constant or the roll stick gain along with its gain scheduling in the roll reference model; however, the roll stick shaping was a design feature that raised questions during the simulation testing and it is believed to be the primary driver of this deficiency. Pilot C felt that the FT task was easy and that there was no tendency for undesirable motions to occur once he settled into the task. Pilot C was asked to repeat this task at the completion of the MRAC flights. His ratings and comments changed significantly (GA 2, FT 2). He commented that it felt like a "nice airplane" and that it had desirable characteristics for both the GA and FT tasks. This change is attributed to the increased separation at which the maneuver was flown, and to the fact that he had flown the task significantly more times with configurations with degraded handling qualities prior to giving this second rating. This training caused him to acclimatize to the heavier stick forces and to adjust his technique accordingly. Despite this adjustment it is still believed that for a more aggressive task the roll forces should be adjusted based on these pilot comments to match the basic F/A-18 airplane control law more closely for follow-on work.

\section{The 2-g Tracking Task}

The 2-g tracking task is a precision air-to-air tracking task. Figure 24 illustrates the maneuver and the pilot cues used in performing this task. The geometry of the task resulted in the test aircraft closing the distance between it and the lead aircraft as the maneuver progressed. The longitudinal range varied from approximately $2500 \mathrm{ft}$ at the start of the maneuver to approximately $1000 \mathrm{ft}$ at the end of maneuver. This range variation worked well in that the GA task was performed with the larger separation and the FT task was performed as the separation closed. The relatively low load factor was chosen due to the limited performance of the vehicle at the slow airspeeds of the limited envelope (see Fig. 4). It does provide, however, a challenging maneuver with well-defined and measureable performance metrics, and it is a task familiar to pilots of fighter aircraft, which makes it a good one for follow-on work with guest pilots. Table 4 contains the performance criteria for the 2-g air-to-air tracking task. 


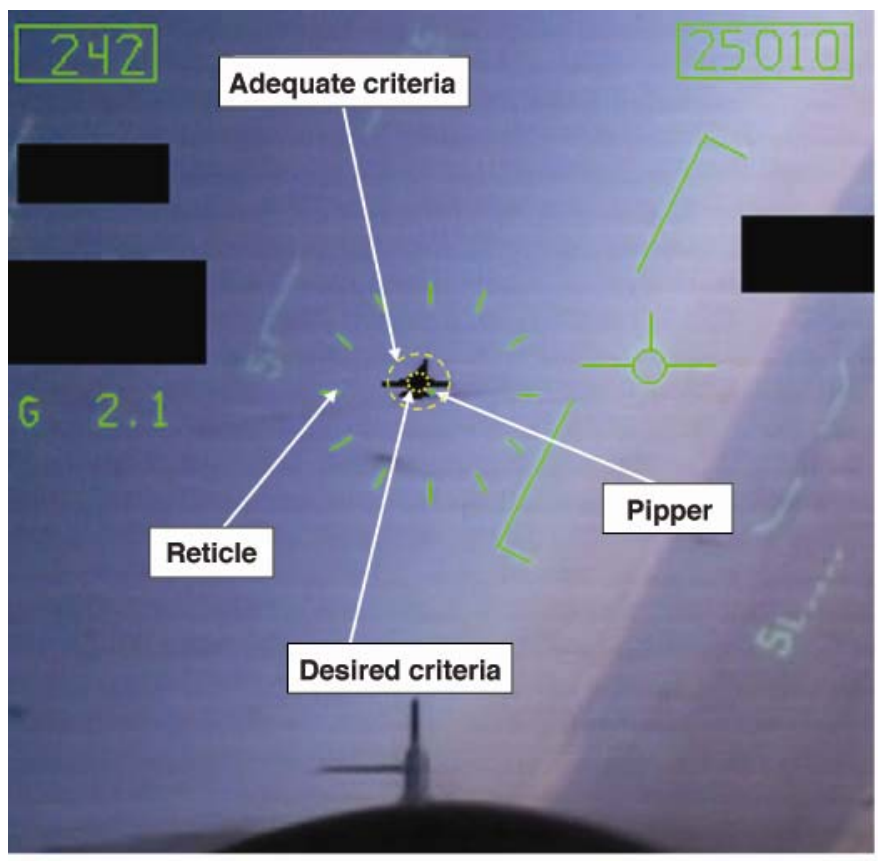

Figure 24. Head-up display view of the 2-g air-to-air tracking task.

Table 4. Performance criteria for the 2-g air-to-air tracking task.

\begin{tabular}{lll}
\hline \hline & Gross acquisition & Fine tracking \\
\hline Desired & Reticle on lead aircraft 0 overshoots & $\begin{array}{l}\text { Pipper on the center of mass of the lead } \\
\text { aircraft } 50 \% \text { of the time } \\
\text { Pipper on the lead aircraft } 50 \% \text { of the time }\end{array}$ \\
\hline
\end{tabular}

Pilot A's comments for the NDI in the 2-g tracking task are similar to his comments for the controller in the in-trail task. In general, his impression was that the NDI and the production control law were very similar. For the GA task he noted that increasing aggressiveness had the effect of causing a pitch bobble, but that he could compensate for that with some shaping of his stick inputs. He commented that this pitch bobble was less pronounced than observed for the production control law. It is believed that this bobble could be eliminated by tailoring the pitch reference model so as to reduce the resonant peak of the pitch frequency response (see Fig. 12) and possibly could be addressed by increasing the natural frequency of the pitch reference model to produce a more crisp pitch response with less perceived lag. In the FT task he commented that the feel was very similar to the production control law. He noted that some undesirable motions were apparent, but that these motions were similar to the production control law and they did not compromise his ability to complete the task. Pilot A repeated this task for the NDI at the end of the MRAC testing, and his ratings were identical to those in table 5; however, he made an additional comment that the roll axis forces were lighter than desirable. This comment about the roll forces contradicts the comments of the other two pilots for the in-trail task, which suggests that a compromise is needed for desirable characteristics for a range of piloting techniques and for different tasks. It also suggests that the gain scheduling of the roll reference model loop gain with angle of attack from Ref. 13 should be revisited to reduce the roll sensitivity for elevated $g$ maneuvers to improve the control harmony for air-to-air tracking tasks. 
Table 5. Cooper-Harper pilot ratings for the 2-g air-to-air tracking task.

\begin{tabular}{lcccc}
\hline \hline & \multicolumn{2}{c}{ Gross acquisition } & \multicolumn{2}{c}{ Fine tracking } \\
\hline & Production & NDI & Production & NDI \\
Pilot A & 4 & 3 & 3 & 4 \\
Pilot B & 3 & 3 & 3 & $2 / 2^{*}$ \\
Pilot C & $3 / 5^{*}$ & $1 / 2^{*}$ & $2 / 3^{*}$ & \\
\hline \hline
\end{tabular}

Pilot B's technique highlighted other interesting aspects of the NDI. He did not notice any tendency for a pitch bobble during the GA task, but commented on undesirable motions in the FT task. These undesirable motions were not apparent to him when flying the task with the production control law. He commented that to get these motions to die out he had to brace his arm against his leg. As noted in Ref. 13, the NDI does not have any stick dead bands. Based on pilot B's comments it is apparent that their omission from the design is causing undesirable rolling motions for maneuvers with relatively large forces in pitch that require little to no roll stick motion. Pilot B did not observe any aggressiveness effects in the GA task but stated that aggressiveness did have an effect on the FT task, which is the opposite of pilot A's comments. Pilot B's opinion and ratings, shown in table 5, were unchanged when he re-flew the task at the conclusion of the MRAC flights, which suggests that his comments and ratings were independent of his familiarity with the task.

Pilot C's comments and his ratings from table 5 suggest that the NDI characteristics fit very well with his piloting techniques and expectations. He commented that the forces seemed a bit heavier than the production control law in both pitch and roll, and that he observed a small pitch bobble at the end of the GA task. He also commented that he felt that the roll axis for the NDI was much more precise than with the production control law. He noted that he did not have to alter his technique to achieve desirable performance with the NDI where he had to be less aggressive with the production control law, especially in the roll axis. He did not comment on any undesirable motion during the fine tracking task.

\section{Summary of Pilot Comments}

The differences in pilot opinion are very useful especially when considered along with each pilot's general approach to the task. Pilot $\mathrm{C}$ tends to be the most aggressive, using high-bandwidth large stick motions to control the aircraft during tight control, and tending to have a high duty cycle with regard to stick motions. Pilot B generally is at the other end of the spectrum in reference to pilot gain. He uses smaller stick motions and attempts to stabilize the stick during the fine tracking portion of each task. Pilot A has been observed in various programs to be in the middle of these two extremes. Knowing this helps to interpret the results and the comments from each pilot. Pilot B tended to find the lack of dead bands to be objectionable for fine tracking, while the other two pilots tended not to mind having to use continuous stick inputs in roll for the air-to-air tracking tasks. Pilot B, however, did not excite the pitch bobble in the GA task regardless of his level of aggressiveness, whereas Pilot $\mathrm{C}$ always noted the bobble regardless of how aggressively he performed the task. Pilot A noticed the bobble when he was aggressive but not when he backed off on his gain. Pilots A and B both commented on the roll axis being slightly more sensitive than desired and that the harmony with the pitch axis could be improved, while Pilot $\mathrm{C}$ commented that both pitch and roll forces were heavy. These opinions suggest that adding dead bands will likely improve pilot B's opinion for FT while not affecting Pilot A or $\mathrm{C}$. The effects of reducing the resonant peak magnitude in the pitch axis while increasing the apparent short-period natural frequency also bear exploring. These changes would likely eliminate the pitch bobble for the GA task for pilots A and C and should improve the control harmony of the pitch and roll axes for pilots A and B. A brief summary of the pilots' comments about the NDI for each task are presented in table 6 . 
Table 6. Summary of pilot comments.

\begin{tabular}{|c|c|c|c|}
\hline & Basic maneuvering & In-trail formation & 2-g tracking \\
\hline Pilot A & $\begin{array}{l}\text { Pitch bobble during pitch } \\
\text { captures, roll control more crisp } \\
\text { than production control for bank } \\
\text { captures, unloads in pitch during } \\
360^{\circ} \text { rolls, roll and pitch } \\
\text { responses faster than production } \\
\text { control law at lower dynamic } \\
\text { pressures }\end{array}$ & $\begin{array}{l}\text { Nearly identical to production } \\
\text { control law with slightly less } \\
\text { undesirable roll motions, } \\
\text { control harmony good, bounded } \\
\text { roll oscillations in GA affected } \\
\text { by aggressiveness }\end{array}$ & $\begin{array}{l}\text { NDI similar to production } \\
\text { control law, pitch bobble in GA } \\
\text { if aggressive but could shape } \\
\text { inputs to eliminate, pitch } \\
\text { bobble less pronounced than } \\
\text { production control law, some } \\
\text { undesirable motions in FT but } \\
\text { similar to production control } \\
\text { law }\end{array}$ \\
\hline Pilot B & $\begin{array}{l}\text { Pitch bobble during pitch } \\
\text { captures, unloads in pitch during } \\
360^{\circ} \text { rolls }\end{array}$ & $\begin{array}{l}\text { Slower roll response than } \\
\text { production control law, } \\
\text { undesirable yaw motions }\end{array}$ & $\begin{array}{l}\text { Undesirable motions during FT } \\
\text { that were not apparent in } \\
\text { production mode and had to } \\
\text { brace arm against leg to damp } \\
\text { them out }\end{array}$ \\
\hline Pilot C & $\begin{array}{l}\text { Hesitation in pitch at FC6, pitch } \\
\text { bobble during pitch captures, } \\
\text { roll response is very precise at } \\
\text { all flight conditions, very } \\
\text { responsive in roll at lower } \\
\text { dynamic pressures relative to } \\
\text { production control law, unloads } \\
\text { in pitch for } 360^{\circ} \text { rolls }\end{array}$ & $\begin{array}{l}\text { Roll forces heavy causing } \\
\text { sluggish response, FT task was } \\
\text { easy with no undesirable } \\
\text { motions }\end{array}$ & $\begin{array}{l}\text { Forces were heavier in both } \\
\text { pitch and roll for NDI than } \\
\text { production controller, small } \\
\text { pitch bobble noted at end of } \\
\text { GA task, roll axis was more } \\
\text { precise than the production } \\
\text { control law, aggressiveness had } \\
\text { no effect on either task whereas } \\
\text { it did for the production control } \\
\text { law }\end{array}$ \\
\hline
\end{tabular}

\section{Conclusions}

The Full-scale Advanced System Testbed platform architecture provides a flexible, robust, and capable environment for testing advanced control concepts. The powerful research processors and envelope protection make rapid prototyping feasible. The short development and testing schedule (four months from design review to flight) for the nonlinear dynamic inversion, which was implemented in the architecture, provides evidence of this fact and affords the controls researcher the flexibility to test novel control elements in a flight environment and to gather valuable real-world data early in the development and testing cycle.

The simulation has been shown to provide good quantitative predictions of stability margins and basic flying characteristics. Some discrepancies were noted in the simulation models, such as a lower stabilator effectiveness in flight than was predicted by the simulation, and some unmodeled time delay in the yaw axis. As a whole, however, the hardware-in-the-loop facility provided a great environment within which to access the control law in order to direct flight experiments and to assure flight safety.

The handling qualities metrics (low order equivalent system, Neal Smith criteria, and bandwidth) evaluated with the flight data provided a means to evaluate the nonlinear dynamic inversion quantitatively. The low order equivalent system analysis highlighted the fact that the flight vehicle had lower short-period natural frequency and damping than the original design but was still in the level 1 region. This analysis shed light on pilot comments about sluggishness and helped to determine the source of a pitch bobble. This conclusion is supported by the bandwidth metric by the fact that the flight data show a lower pitch bandwidth than that predicted by the simulation, causing a small but noticeable degradation in pitch handling qualities. The Neal Smith criteria highlighted a higher-than-predicted sensitivity to pilot control bandwidth which, again, contributed to the pitch bobble noticed during aggressive pitch tasks.

The flight environment produced a wealth of pilot comments that highlighted the real characteristics of the nonlinear dynamic inversion, and shed light on questions left unanswered by simulation tests. In the flight environment the pilots tended to have different aggression levels than in the simulation, and reacted to different cues such as the real vehicle motion. All three pilots found the unload tendency for the $360^{\circ}$ rolls to be objectionable in flight, whereas in the simulation there was no mention of this deficiency. In the simulation there were no comments about the increased stick forces for the nonlinear dynamic inversion nor any comments about control harmony 
issues; however, all three pilots had significant comments about the harmony between the pitch and roll axes and the stick forces for both of the closed-loop tracking tasks. The different piloting techniques revealed the need for both dead bands in the pitch and roll stick as well as a need to modify both the roll stick shaping and the roll stick gain scheduling with angle of attack. Additionally, the small discrepancy in pitch surface effectiveness had a noticeable effect on the pitch handling qualities, and warrants some small design changes prior to follow-on work. This pitch deficiency was found to be sensitive to pilot aggressiveness and technique, such that more aggressive techniques tended to exacerbate the undesirable pitch motions. The pilot comments could be readily tied to specific design elements within the control law and their quantitative effect assessed by the results of the handling qualities metrics from flight data. The simulation testing, while being relatively accurate from a quantitative standpoint (with a few exceptions), did not uncover most of the valuable insight gained from the brief flight-test program.

The baseline control law was found to have desirable handling qualities with a few noteworthy deficiencies. It was found to be a flexible architecture that can produce very good handling qualities within a short design cycle: pilot comments about this intuitive architecture can quickly be addressed with simple design modifications. The control law was found to be robust to differences between simulation models and the actual flight characteristics of the vehicle. This control law will provide a good baseline control law for future controls research into adaptive controls and the control of flexible structures among other advanced control techniques in a flight environment.

\section{References}

${ }^{1}$ Balas, G. J., “Flight Control Law Design: An Industry Perspective,” European Journal of Control, (2003)9, pp. $196-215$.

${ }^{2}$ Bordignon, K., and Bessolo, J., “Control Allocation for the X-35B,” AIAA-2002-6020, 2002.

${ }^{3}$ Hanson, C., Schaefer, J., Burken, J. J., Johnson, M. A., and Nguyen, N., "Handling Qualities Evaluations of Low Complexity Model Reference Adaptive Controllers for Reduced Pitch and Roll Damping Scenarios,” AIAA-2011-\#\#\#\# (to be published), 2011.

${ }^{4}$ Schaefer, J., Hanson, C., Johnson, M.A., and Nguyen, N., "Handling Qualities of Model Reference Adaptive Controllers with Varying Complexity for Pitch-Roll Coupled Failures,” AIAA-2011-\#\#\#\# (to be published), 2011.

${ }^{5}$ Steinberg, M. L., “A Comparison of Intelligent, Adaptive, and Nonlinear Flight Control Laws,” AIAA-99-4044, 1999.

${ }^{6}$ Kaneshige, J., Bull, J., and Totah, J. J., “Generic Neural Flight Control and Autopilot System,” AIAA-2000-4281, 2000.

${ }^{7}$ Rysdyk, R. T., and Calise, A. J., "Fault Tolerant Flight Control Via Adaptive Neural Network Augmentation," AIAA-98-4483, 1998.

${ }^{8}$ Bosworth, J. T., "Flight Results of the NF-15B Intelligent Flight Control System (IFCS) Aircraft with Adaptation to a Longitudinally Destabilized Plant,” AIAA-2008-6985, 2008.

${ }^{9}$ Bosworth, J. T., and Williams-Hayes, P. S., "Flight Results from the NF-15B Intelligent Flight Control System (IFCS) Aircraft with Adaptation to a Simulated Stabilator Failure,” AIAA-2007-2818, 2007.

${ }^{10}$ Yedavalli, R. K., Shankar, P., and Doman, D. V., "Robustness Study of the Dynamic Inversion Based Indirect Adaptive Control of Flight Vehicles with Uncertain Model Data,” Proceedings of the 2003 American Control Conference, 2003, pp. 1005-1010.

${ }^{11}$ Frost, S. A., Taylor, B. R., Jutte, C. V., Burken, J. J., Trinh, K. V., and Bodson, M., “A Framework for Optimal Control Allocation with Structural Load Constraints,” AIAA-2010-8112, 2010.

${ }^{12}$ Gregory, I. M., “Modified Dynamic Inversion to Control Large Flexible Aircraft - What’s Going On?” AIAA-99-3998, 1999.

${ }^{13}$ Miller, C. J., "Nonlinear Dynamic Inversion Baseline Control Law: Architecture and Performance Predictions," AIAA-2011-\#\#\#\# (to be published), 2011.

${ }^{14}$ Clarke, R., Allen, M. J., Dibley, R. P., Gera, J., and Hodgkinson, J., "Flight Test of the F/A-18 Active Aeroelastic Wing Airplane,” AIAA-2005-6316, 2005.

${ }^{15}$ Bosworth, J. T., and West, J. C., "Real-Time Open-Loop Frequency Response Analysis of Flight Test Data," AIAA-86-9738, 1986.

${ }^{16}$ U.S. Department of Defense, Flying Qualities of Piloted Aircraft, MIL-STD-1797B, 15 February 2006.

${ }^{17}$ Mitchell, D. G., and Field, E. J., "Nonlinearities and PIO with Advanced Aircraft Control Systems," RTO Meeting Proceedings 51 (RTO-MP-051), 2001, pp. 28-1-28-14.

${ }^{18} \mathrm{Neal}, \mathrm{T}$. P., and Smith, R. E., “An In-Flight Investigation to Develop Control System Design Criteria for Fighter Airplanes,” AFFDL-TR-70-74, Volume I, Air Force Flight Dynamics Laboratory, December 1970.

${ }^{19}$ Hoh, R. R., Mitchell, D. G., and Hodgkinson, J., "Bandwidth: A Criterion for Highly Augmented Airplanes,” AGARD Criteria for Handling Qualities of Mil. Aircraft, 1982.

${ }^{20}$ Hodgkinson, J., and LaManna, W. J., "Equivalent System Approaches to Handling Qualities Analysis and Design Problems of Augmented Aircraft,” AIAA-1977-1122, 1977.

${ }^{21}$ Wood, J. R, and Hodgkinson, J., "Definition of Acceptable Levels of Mismatch for Equivalent Systems of Augmented CTOL Aircraft,” MDC A6792, McDonnell Aircraft Company, St. Louis, Missouri, 1980.

${ }^{22}$ Gray, W. R., III, “Handling Qualities Evaluation at the USAF Test Pilot School,” AIAA-2009-6317, 2009.

${ }^{23}$ Cooper, G. E., and Harper, Jr., R. P., “The use of Pilot Rating in the Evaluation of Aircraft Handling Qualities,” NASA TN D-5153, 1969. 


\title{
Nonlinear Dynamic Inversion Baseline
}

Control Law: Flight-Test Results for the Full-scale Advanced Systems Testbed F/A-18 Airplane

\author{
Chris Miller \\ Dryden Flight Research Center \\ Flight Control and Dynamics \\ 661-276-2902 \\ Chris.j.miller@nasa.gov
}




\section{Outline}

- Research Objectives

- Full-Scale Advanced Systems Testbed (FAST) Background

- Control Law Description

- Flight Results

- Open loop pitch frequency response

- Closed loop pitch reference model tracking

- Pitch handling qualities metrics

- Pilot comments and ratings for closed loop tasks

- Conclusions

- Questions 


\section{Motivation for NDI}

Research Objectives

- RFI distributed in 2009 for research related to resilient aircraft control

- RFI responses discussed at AIAA GNC in 2009

- FAA, Academia, NASA, and Industry

- Three areas highlighted

- Investigate simple yet effective adaptive algorithms

- Explore pilot interactions with adaptive controllers

- Incorporate structural feedback into the flight control system
Why nonlinear dynamic inversion

- Utilized in literature for baseline control law for a number of adaptive and structural control techniques

- Explicit model following

- NDI contains explicit reference models, which integrate nicely with many adaptive control techniques, such as model reference adaptive control

- Failure modeling

- Fundamental-level failures (uncertainty in elements of the A- and B-matrices) can be modeled by altering the NDI reference models and PI compensators, and onboard aerodynamic tables

- Analysis formulation

- The NDI architecture is easy to analyze and include in stability proofs

- Open source architecture

- NDI is an open-source architecture. By specifying non-ITAR reference models, simulation and flight test data can be openly published 


\section{FAST Vehicle}

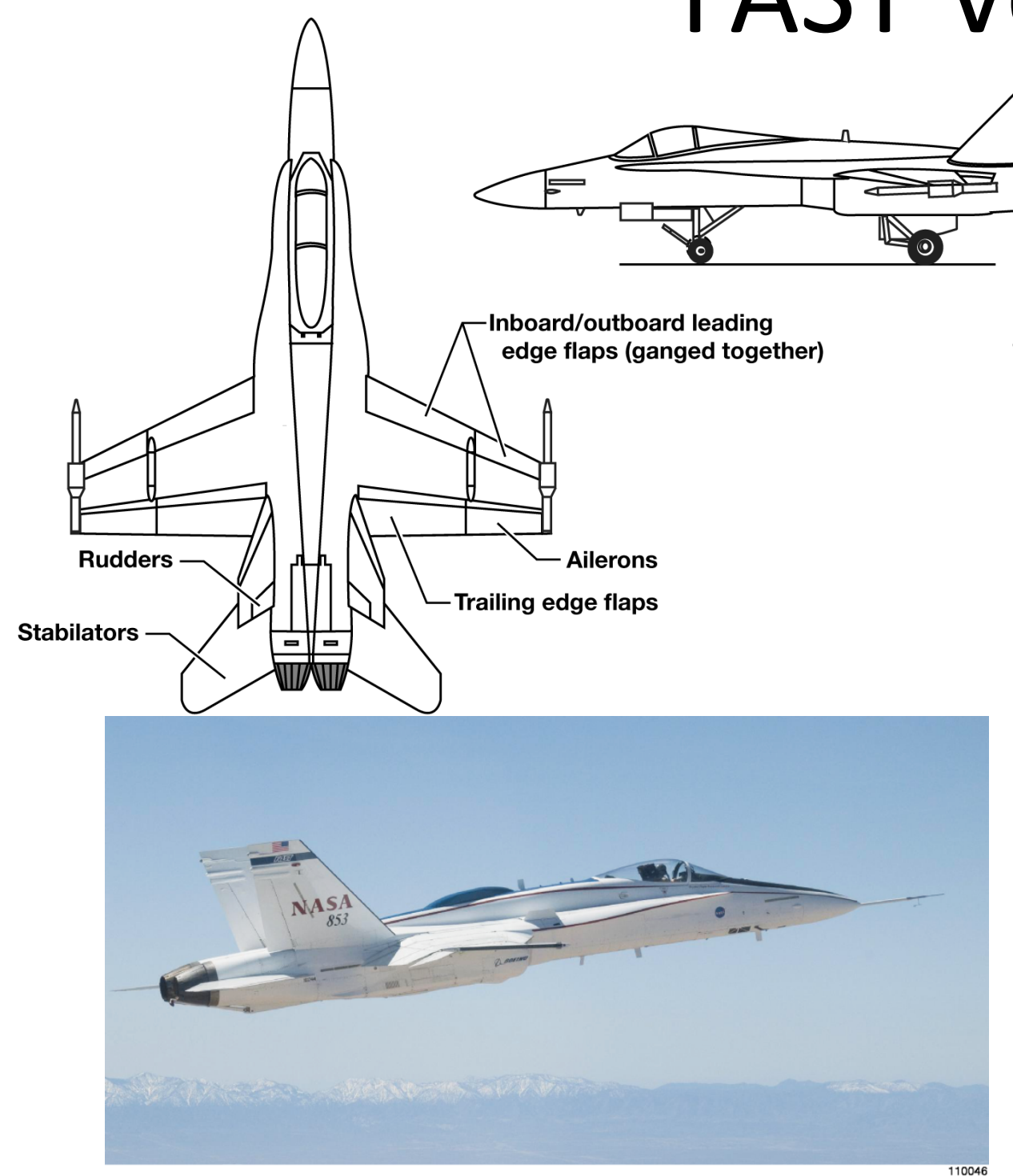

- Modified F/A-18

- Research flight control computers

- Quad RFCS

- Dual ARTS

$\overline{110047}_{0}$ Research instrumentation

- Research EGI

- Static and dynamic structural instrumentation

- FDMS

- Strain

- Accelerometers

- Nose boom 


\section{FAST Hardware Background}

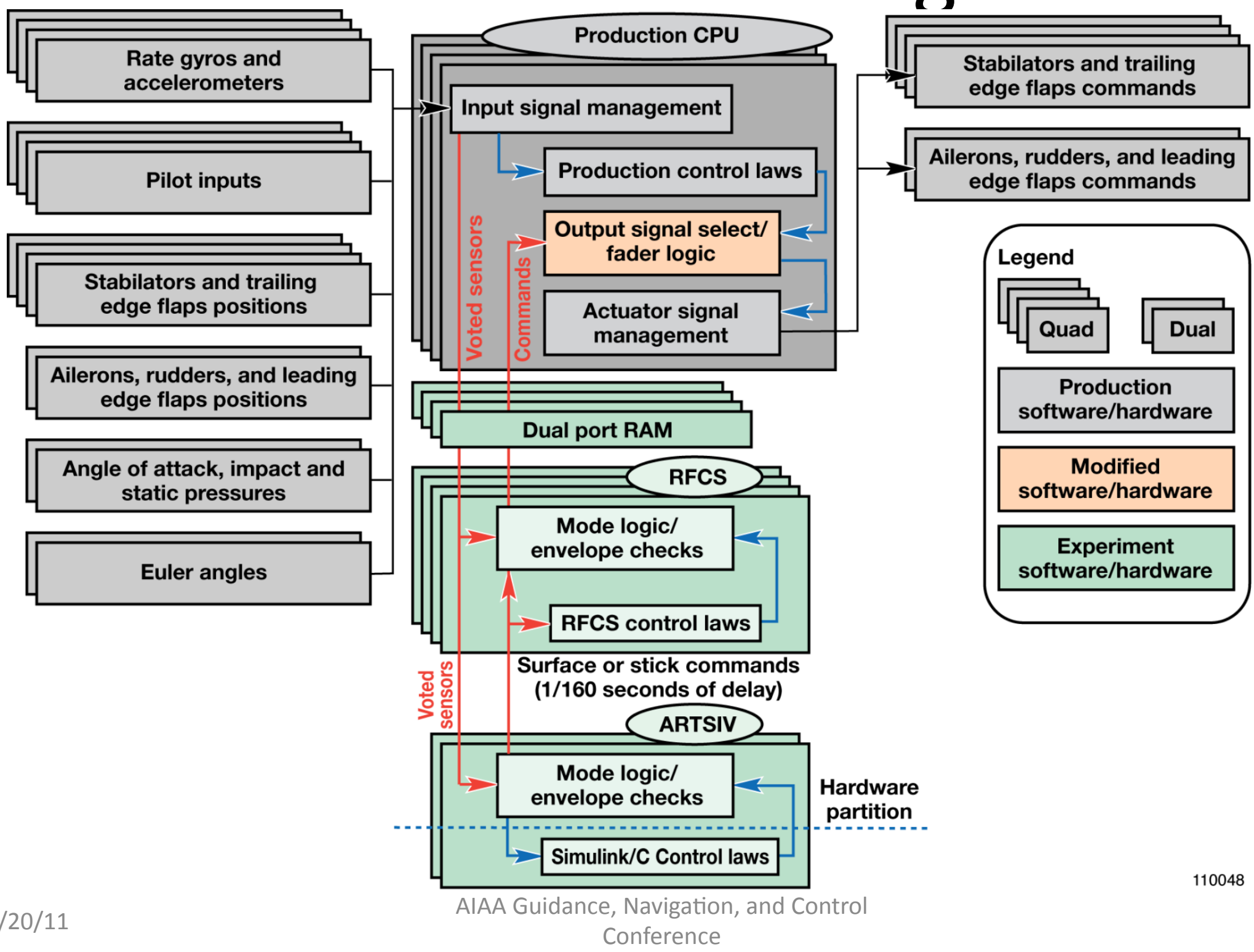




\section{FAST Envelope}

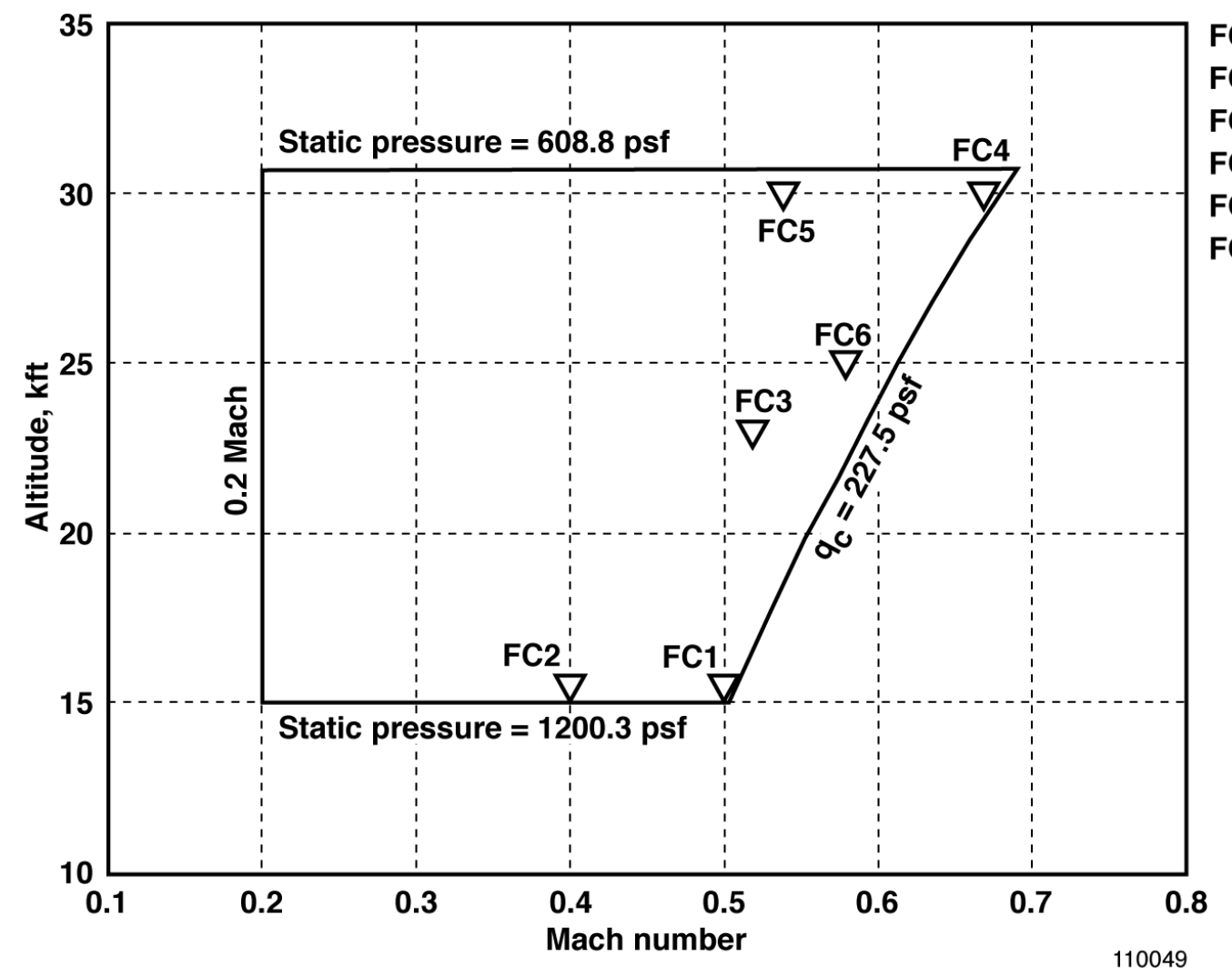

FC1: 250 KCAS, 15.5 kft FC2: 200 KCAS, $15.5 \mathrm{kft}$ FC3: $223 \mathrm{KCAS}, 23.0 \mathrm{kft}$ FC4: $250 \mathrm{KCAS}, 30.0 \mathrm{kft}$ FC5: $200 \mathrm{KCAS}, 30.0 \mathrm{kft}$ FC6: 240 KCAS, $25.0 \mathrm{kft}$ 
Fes

\section{Control Law Background}




\section{Control Law Overview}

- 5 Major Components

- Dynamic Inversion

- Computes surface positions to produce desired dynamics

- Conservation of angular momentum formulation

- Aerodynamic Tables with Control Mixing

- Tabulates control surface effectiveness, and manages surface usage priorities

- Simplified F/A-18 simulation aerodynamic tables

- Reference Models

- Compute desired vehicle dynamics from pilot commands

- Standard first and second order transfer functions for angular rates

- PI Compensator

- Adds robustness and disturbance rejection

- Tuned to match reference model poles

- Structural Filters

- Prevent undesirable ASE effects

- Unmodified from production filters

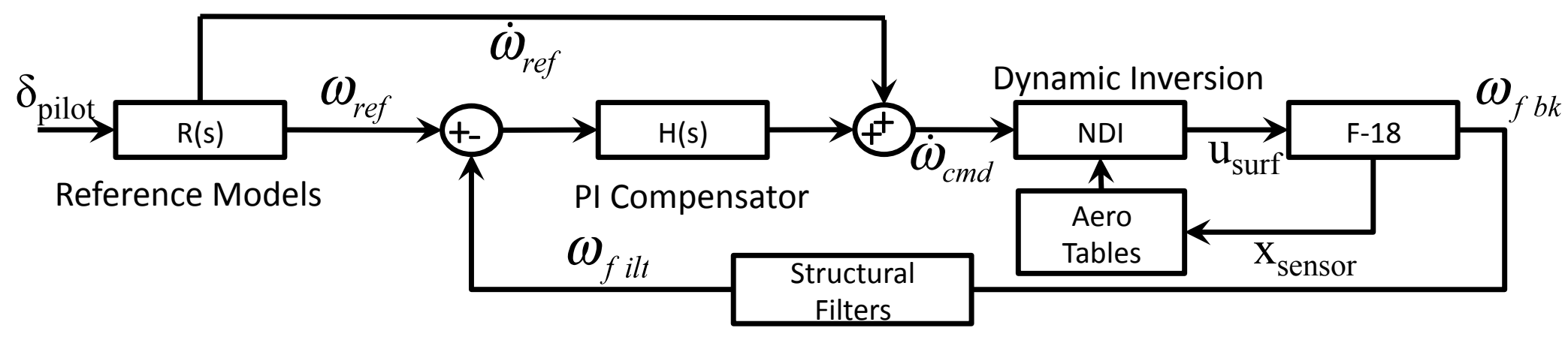


F每区

Flight Results 


\section{Open Loop Pitch Response}

- Open loop response reconstructed from closed loop

- Open loop gain behavior

- Constant offset suggests loop gain difference

- Matches effect of the simulation over predicting stabilator effectiveness

- Increases phase and gain margins

- Open loop phase behavior

- Well predicted by simulation

- Suggests all sources of time delay and lag are well modeled
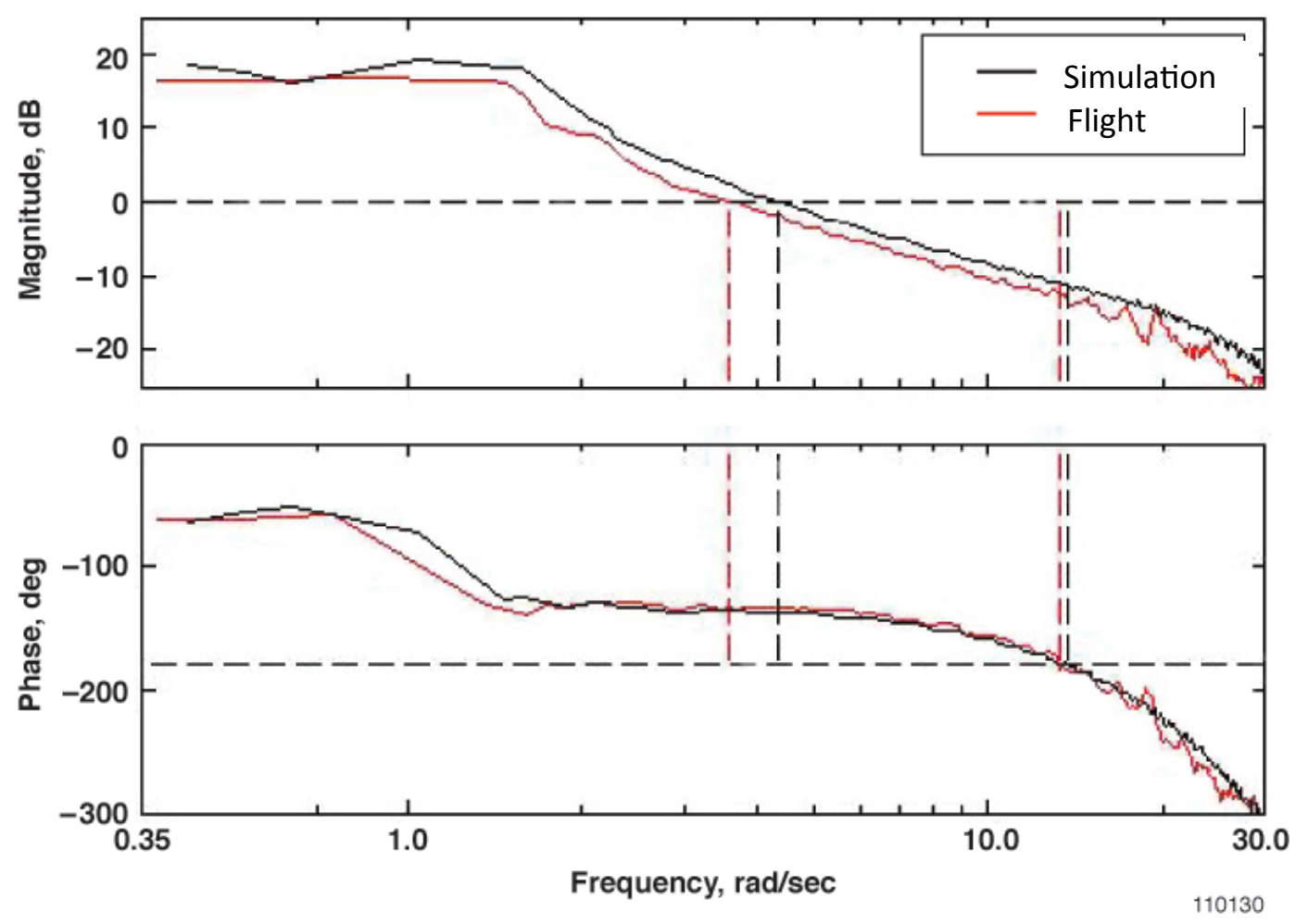


\section{Closed Loop Pitch Response}

- Primary effect of over prediction of stabilator effectiveness apparent in closed loop gain response

- Reduced apparent short period natural frequency

- Causes high frequencl response to lie outsidı

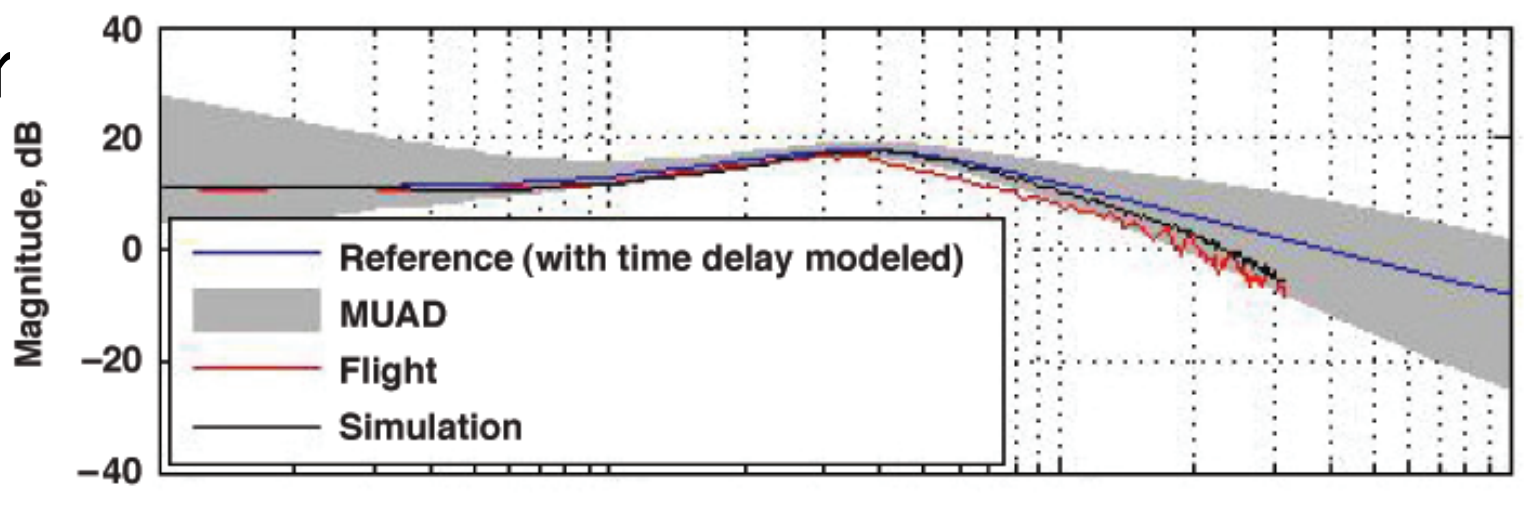
of MUAD

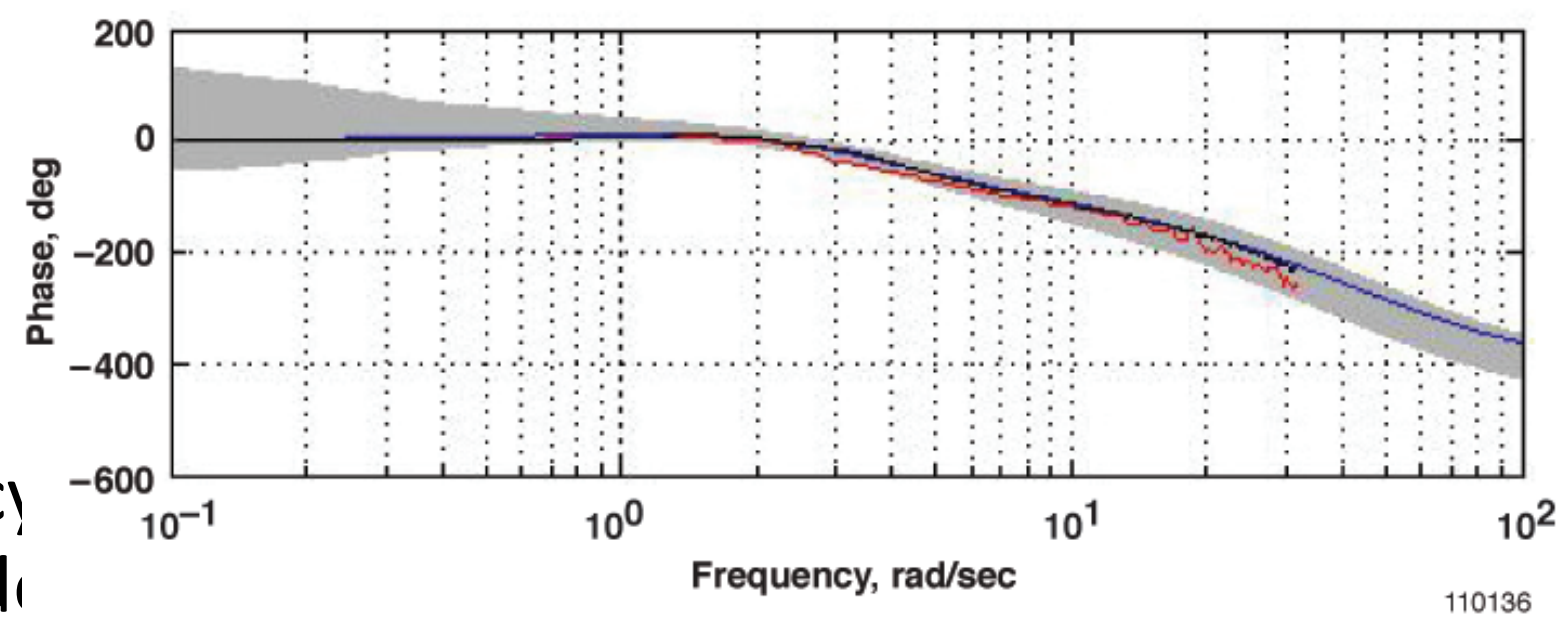




\section{Low Order Equivalent Systems}

- Short period approximation

- Reduced natural frequency

- Stabilators less effective than predicted

- Damping ratio well predicted but lower than desired

- Uncompensated actuator dynamics

- Equivalent time delay well predicted but higher than desired

- Actuator, and sensor dynamics

- Structural filters

- Architecture transport delay
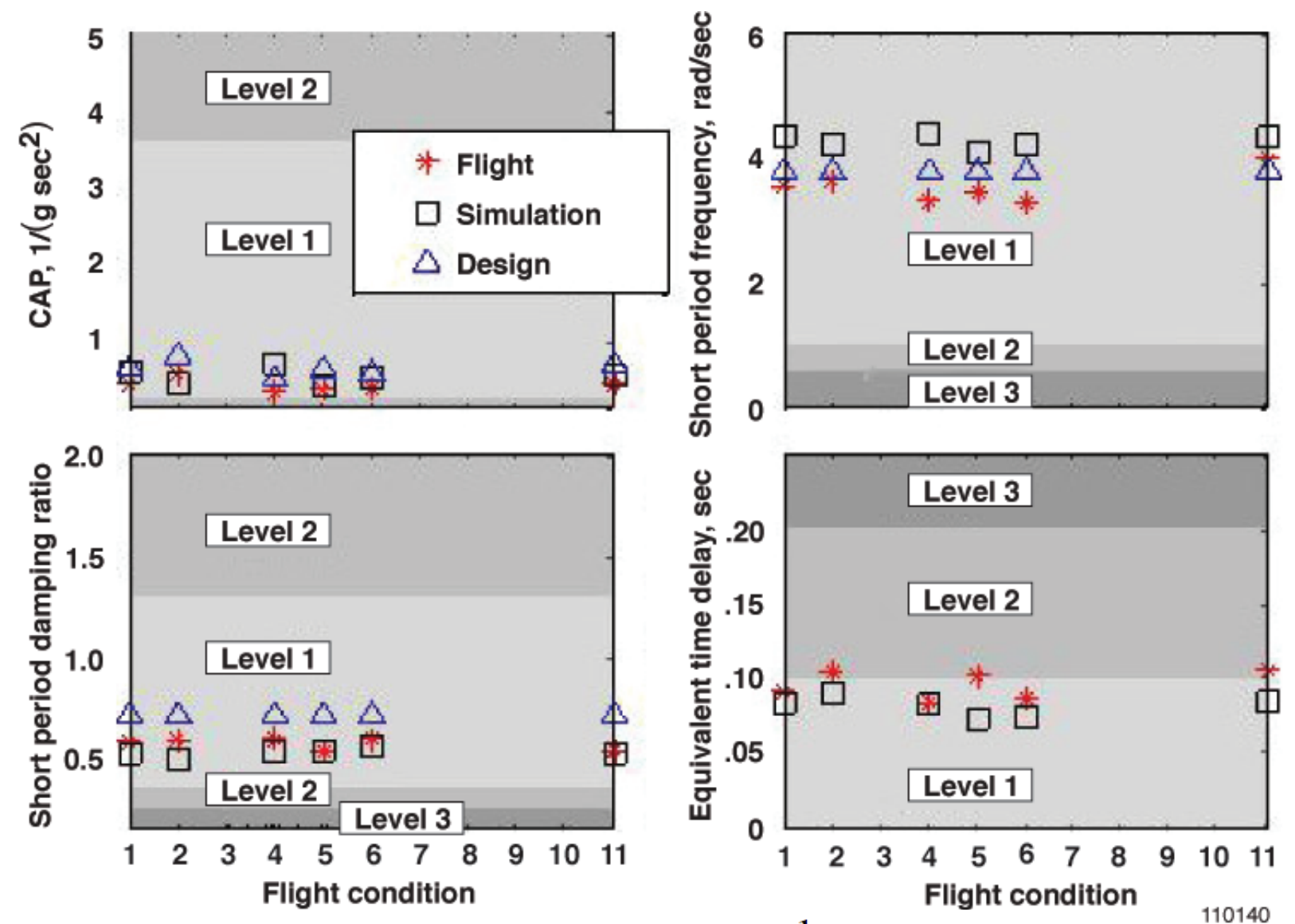

$$
\frac{\mathrm{q}}{\mathrm{F}_{\mathrm{s}} \text { or } \delta_{\mathrm{s}}}=\frac{\mathrm{K}_{\mathrm{q}_{\mathrm{e}}}\left(\mathrm{s}+\frac{1}{\mathrm{~T}_{\theta_{2}}}\right) \mathrm{e}^{-\tau_{\theta_{e}} s}}{\left(\mathrm{~s}^{2}+2 \zeta_{\mathrm{sp}_{\mathrm{e}}} \omega_{\mathrm{sp}_{\mathrm{e}}} \mathrm{s}+\omega_{\mathrm{sp}_{\mathrm{e}}}^{2}\right)}
$$




\section{Neal-Smith Analysis}

- Based on a tuned mathematical compensator for a desired pitch attitude frequency response

- Flight to Simulation Comparison

- Flight handling qualities more sensitive to increased bandwidth

- More lead compensation required for increased bandwidth

- Resonant peak grows more rapidly with increasing bandwidth

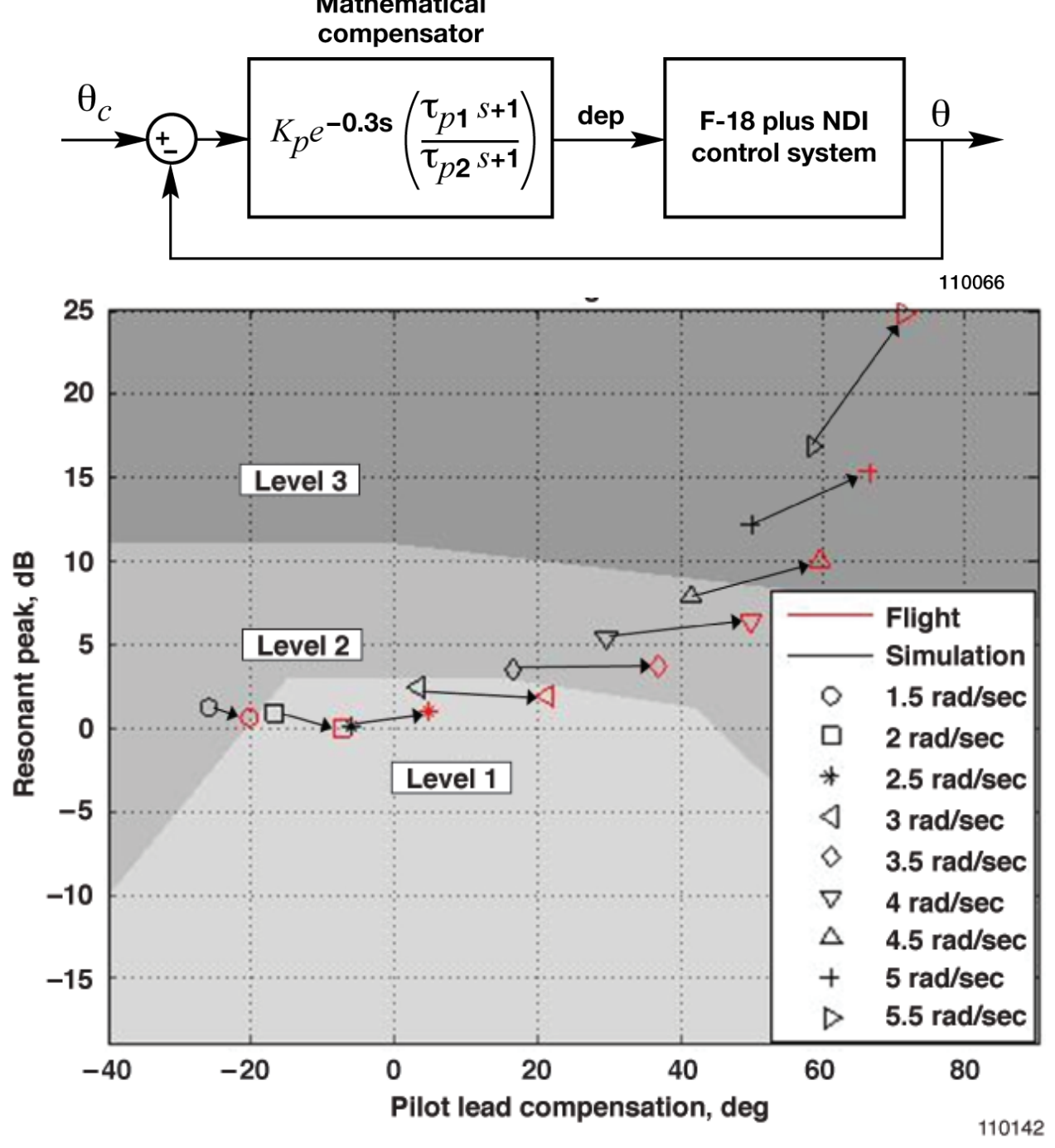


F每区

\section{Piloted Evaluations}




\section{Two g Air-to-Air Tracking Task}

performed at FC6 (240 KCAS, $25 \mathrm{kft}$ )

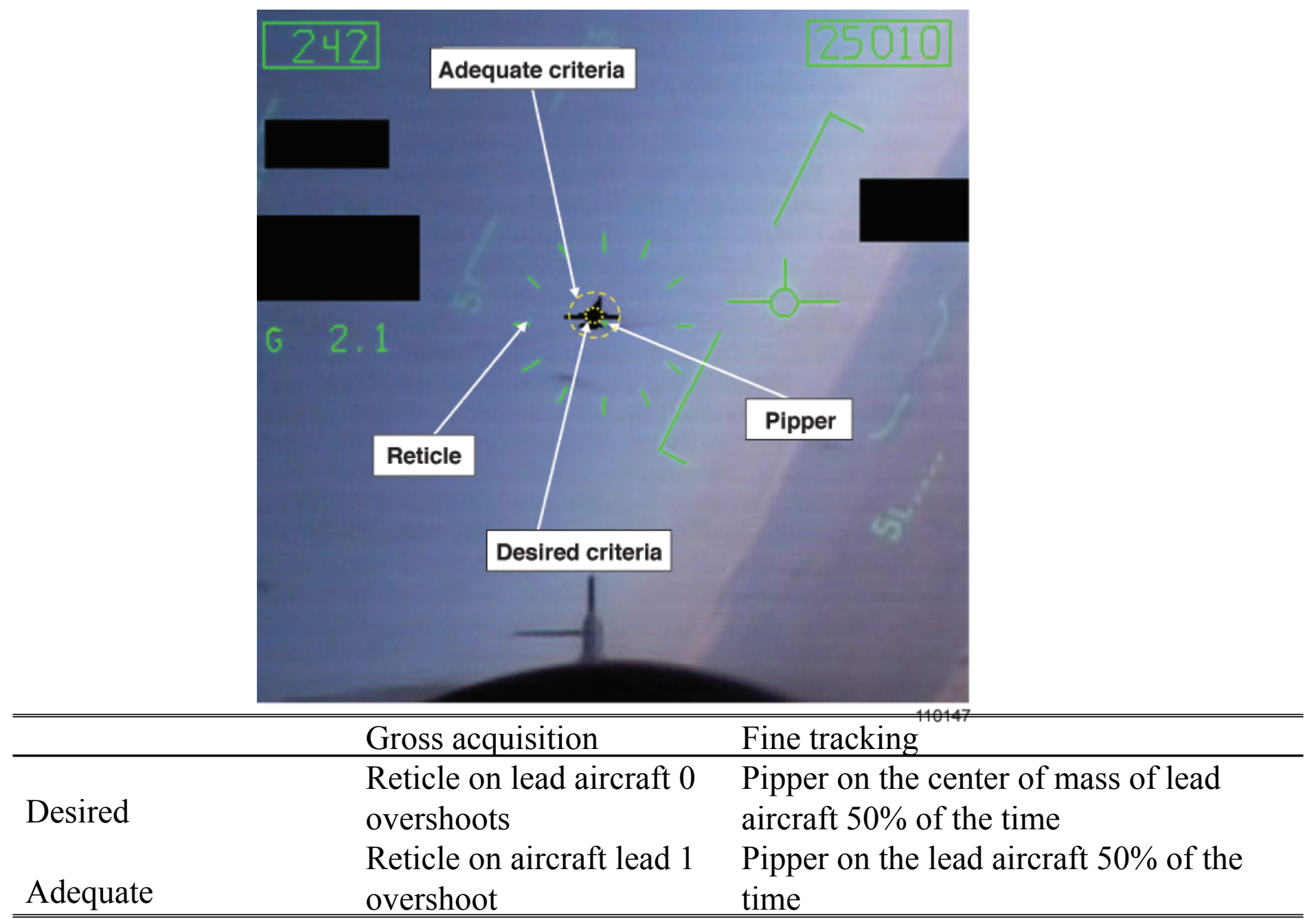




\section{In-Trail Formation Task}

performed at FC6 (240 KCAS, $25 \mathrm{kft}$ )

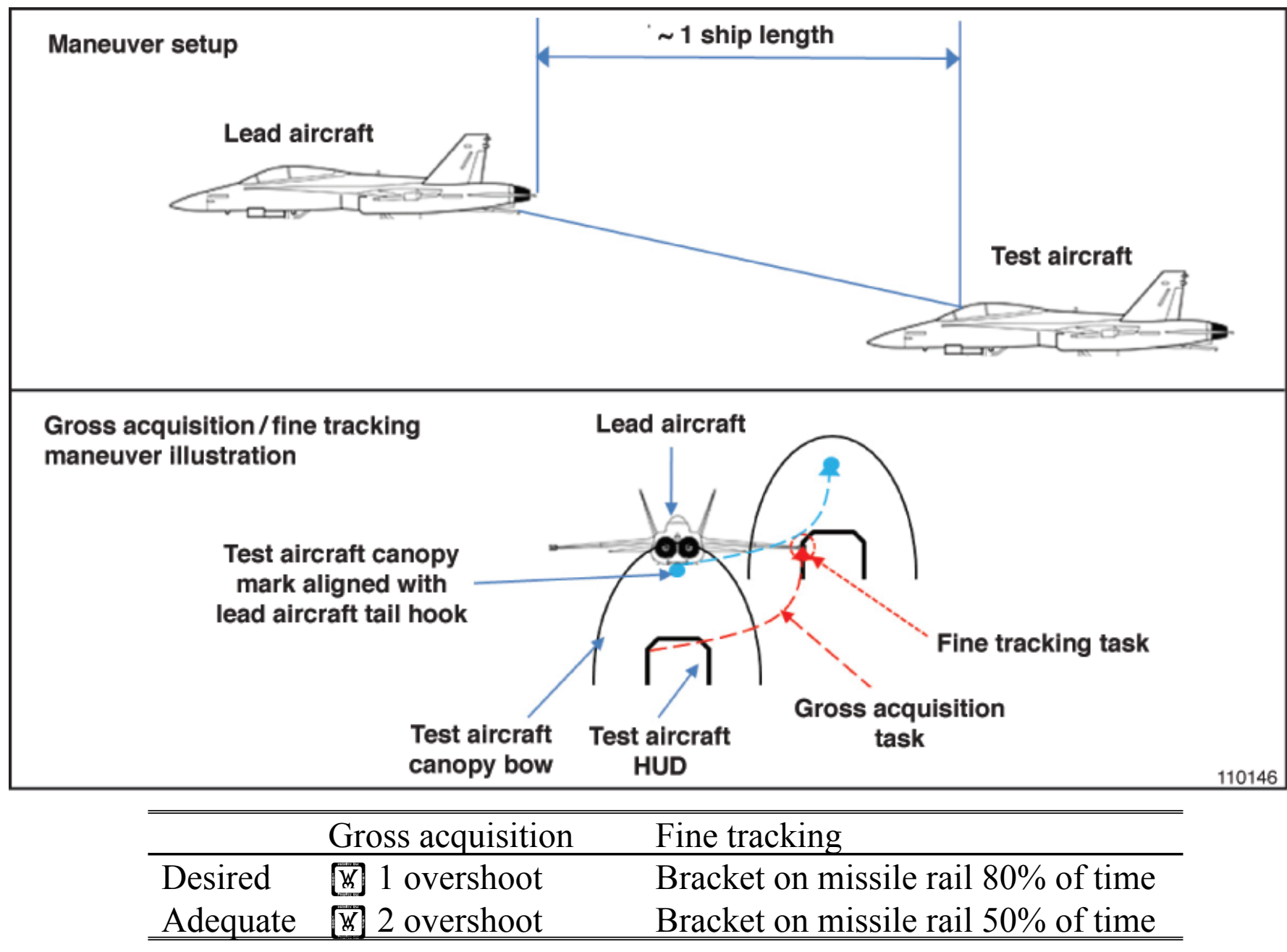




\section{Pilot Ratings}
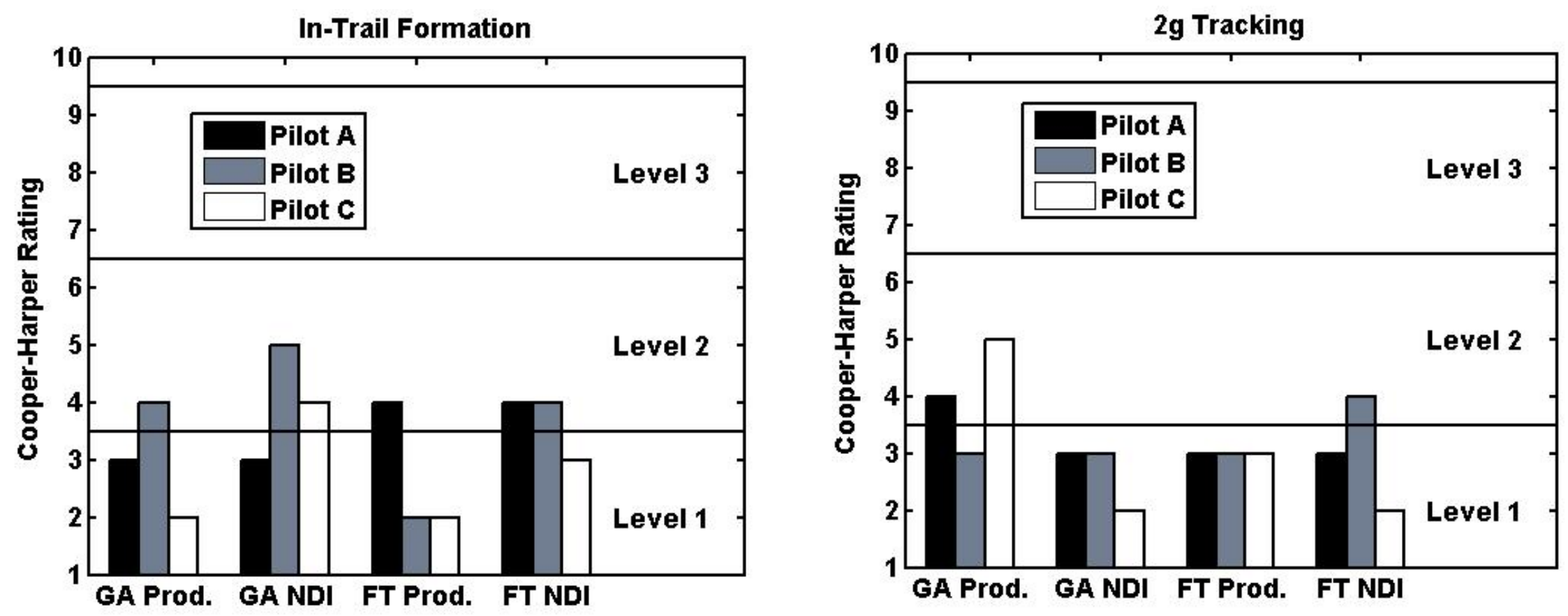


\section{Summary of Pilot Comments}

- Basic maneuvering

- Pitch bobble apparent for high bandwidth pitch captures

- Roll response more precise (crisp) than production control law especially noticeable at lower dynamic pressures

- Undesirable unload for 360 $\mathrm{W}$. rolls (easily addressed with an additional feed forward pitch rate command)

- In-trail formation task

- Roll forces heavier than production control law

- Two g tracking task

- Largest difference in pilot opinions (related to aggressiveness)

- Low gain pilot commented on undesirable motions during fine tracking (related to a lack of stick dead bands)

- High gain pilot found the more precise roll response highly desirable, and found the higher pitch and roll stick forces to be beneficial 


\section{Conclusions}

- The FAST architecture has been shown to provide an environment where control experiments can be brought to flight quickly

- NDI completed in 4 months

- The simulation was found to provide good qualitative results, but did not yield the most valuable data with regard to pilot comments

- The handling qualities metrics were found to be very valuable in explaining pilot comments quantitatively

- Pitch bobble and sluggishness related to pilot bandwidth and short period properties

- NDI specific conclusions

- With some compensation good handling qualities can be achieved with this very simple architecture

- Most of the negative comments were related to design features that are easily tunable

- Stick dead bands needed, along with pitch rate compensation during 360 网 rolls to eliminate undesirable motions, roll stick shaping modification for lighter roll forces

- NDI provides a good baseline control law upon which advanced control elements (adaptive, structural control) can be built and taken to flight quickly 


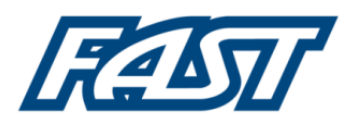

\section{Questions}

